\title{
PILE-UP SOLUTIONS FOR SOME SYSTEMS OF CONSERVATION LAWS MODELLING DISLOCATION INTERACTION IN CRYSTALS*
}

\author{
A. CARPIO $^{\dagger}$, S. J. CHAPMAN ${ }^{\ddagger}$, AND J. L. L. VELÁZQUEZ ${ }^{\dagger}$
}

\begin{abstract}
Some continuum models for dislocation interactions in a simple crystal geometry are studied. The simplest models are mixed systems of conservation laws which are shown to exhibit singularities and instabilities. These are then regularized, leading to parabolic free-boundary problems. In both cases, solutions describing the formation of structures such as dislocation pile-ups are discussed.
\end{abstract}

Key words. dislocation dynamics, degenerate systems of conservation laws, free-boundary problems, pattern formation

AMS subject classifications. 35R $35,35 \mathrm{~L} 65,35 \mathrm{~K} 65,35 \mathrm{~L} 80$

PII. S0036139999364347

1. Introduction. In this paper we are concerned with the study of dislocations, which are line defects in an elastic crystal. Let us first recall some of their wellknown properties (cf. [3], [12]). When a sufficiently large stress is applied, these dislocations glide along the crystallographic planes of the crystal and interact with other dislocations they find on their way. In addition, new dislocations are observed to be generated at certain nucleation sites. As a result they appear typically in very large numbers $\left(10^{12}\right.$ dislocations $/ \mathrm{cm}^{2}$ in heavily worked metals) and modify the mechanical properties of the material. In particular, dislocations are thought to control the plastic properties of crystalline solids (at low temperature).

It is well known that, under an applied stress, crystals deform elastically up to a critical value of this stress, known as the yield stress. For higher stresses, the deformation becomes plastic (irreversible) and ends up eventually in fracture. The yield stress is thought to be the stress at which large numbers of dislocations start moving. Once in the plastic regime, the generation, motion, and interaction of dislocations results in the formation of complicated networks of defects in the microscopic structure of the material. When these networks are so dense that dislocations cannot move freely, the crystal hardens (work hardening). This effect is very important when working with metals, since heavily worked metals are stronger than unworked metals.

Dislocations can be described in many different ways, depending on the lengthscale on which they are viewed. At the microscopic level, they appear as defects in the crystalline lattice. Then, if the separation between dislocations is not too small, there is a mesoscopic scale at which the dislocations may be modelled as line singularities of the elastic stress evolving in a continuous material [6]. Finally, at a macroscopic scale containing large numbers of dislocations we can think in terms of a continuous dislocation density.

* Received by the editors November 22, 1999; accepted for publication (in revised form) December 15, 2000; published electronically May 16, 2001.

http://www.siam.org/journals/siap/61-6/36434.html

$\dagger$ Departamento de Matematica Aplicada, Universidad Complutense, Madrid 28040, Spain (ana_carpio@mat.ucm.es, velazque@sunma4.mat.ucm.es). The work of the first author was partially supported by the Spanish-British integrated action HB1997-0162. The research was also supported by the European TMR on Hyperbolic Systems ERB-FMRXCT-960033. The research of the third author was supported by DGES grant PB96-0614.

${ }^{\ddagger}$ Oxford Center for Industrial and Applied Mathematics, Mathematical Institute, University of Oxford, 24-29 St. Giles, Oxford OX1 3LB, UK (chapman@maths.ox.ac.uk). 
At the microscopic level, models for the evolution of the particles can be obtained writing the laws of motion for each particle of the lattice, choosing suitable interaction potentials. Many numerical simulations of these types of models have been carried out (see, for instance, [4], [14], [17]). At the mesoscopic scale, models for the evolution of the line singularities can be written down and rules chosen for their interaction. (Ideally these rules would come from an analysis of the microscopic scale through some asymptotic limit; such an analysis has yet to be carried out successfully, and laws of motion and interaction are often taken from experimental observations.) Such models have been simulated numerically in [7].

One goal of this paper is to formulate models for the dynamics of macroscopic densities of dislocations. Several models involving densities of dislocations in different frameworks have been developed in [1], [11], [13]. We are concerned here with a common process experimentally observed in worked materials that is the formation of a honeycomb of regions of high and low dislocation density, as dislocations on different slip planes interact and tangle up, effectively pinning each other. One of our prime motivations in this work is to try to determine the simplest model of dislocation interaction which will exhibit this pattern formation.

We introduce two types of models. The first are systems of conservation laws of changing type (they may be either strictly hyperbolic, or nonstrictly hyperbolic, or elliptic in different regions). The onset of nonstrictly hyperbolic regions creates a number of difficulties, since, from the mathematical point of view, the initial value problem may be ill-posed.

The second are regularizations of these systems by terms involving higher derivatives, which we expect to have a smoothing effect. In this case we are led to the study of free-boundary problems with vanishing dissipation.

Despite their simplicity our models exhibit instabilities due to the loss of strict hyperbolicity in the equations, which should lead to such pattern formation. They can also describe some typical dislocation patterns, such as dislocation pile-ups. We find that these structures can arise when a moving family of dislocations meets a high density of some other family of dislocations on its way. This "barrier" of dislocations makes glide difficult so that the moving dislocations get stuck and pile up.

This paper is organized as follows. In the next section we propose the models. In section 3, we study some solutions showing pile-up formation for the degenerate systems of conservation laws by methods of characteristics. In section 4 we construct pile-up solutions for the regularized models by solving rigorously some free-boundary problems with small viscosity . We also study their limiting behavior as the regularizing terms tend to zero in section 5 using formal asymptotics. Finally, some technical points are analyzed in a detailed way in several appendices at the end of the paper.

2. The models. Details of the structure of dislocations can be found in any book on the field (see, for instance, [3], [12]). In general, they may be characterized by their tangent vector and a microscopic parameter known as the Burgers vector, which measures the form of the local mismatch in the crystal lattice. ${ }^{1}$ The canonical cases for rectilinear dislocations are to have the Burgers vector parallel to the tangent line (screw dislocations) or perpendicular to it (edge dislocations). More complex curved dislocations can be described as combinations of these elementary types.

\footnotetext{
${ }^{1}$ If we think of the dislocation as being created by a cut-and-weld operation, that is, by slicing a perfect crystal along a semi-infinite plane bounded by the dislocation line, moving the material on one side of this plane relative to the other, and then welding the two sides together again, then the Burgers vector gives the relative displacement of the two halves before rejoining.
} 


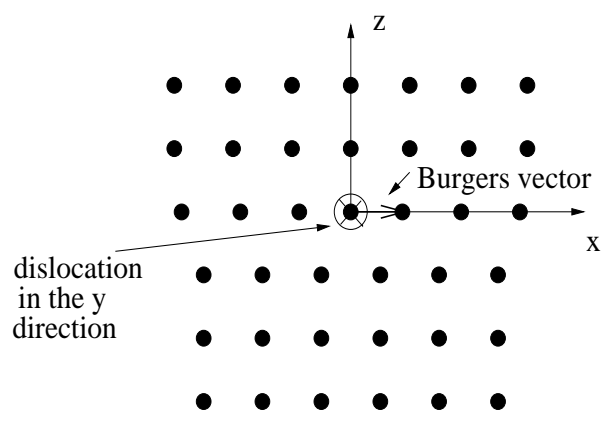

FIG. 2.1. Edge dislocation.

Typically, it is energetically more favorable for a dislocation to "glide" in the slip plane spanned by its tangent and Burgers vectors, rather than to "climb" out of this plane [3]. In this paper, since we are looking at very simple models, we will restrict our analysis to the interaction of edge dislocations in a cubic crystal. In Figure 2.1 we show a cross section of a typical edge dislocation, along with its Burgers vector.

We obtain a model for the interaction of two families of edge dislocations. We take the first family to be tangent to the $z$-direction and Burgers vector in the $x$-direction, and the second family to have tangent in the $y$-direction and Burgers vector in the $x$-direction. Thus, the first family has the $x z$-plane as its slip plane, while the second family has the $x y$-plane as its slip plane, and if we assume that the dislocations remain rectilinear then both families will glide in the $x$-direction [3]. We refer to them as "dislocations type 1" and "dislocations type 2," respectively. By symmetry considerations, the problem can be reduced to a one-dimensional problem (see [5] for more details on the modelling), giving two populations with densities $w_{1}(x, t)$ and $w_{2}(x, t)$, respectively. We want to determine how these density profiles evolve with time.

Conservation of dislocations for both families yields

$$
\begin{aligned}
& \frac{\partial w_{1}}{\partial t}+\frac{\partial}{\partial x}\left(w_{1} v_{1}\right)=0 \\
& \frac{\partial w_{2}}{\partial t}+\frac{\partial}{\partial x}\left(w_{2} v_{2}\right)=0
\end{aligned}
$$

where $v_{i}$ is the velocity of family $i$.

To close the model we need a law relating the speed of the dislocations to the external forces and the densities.

Dislocations move in their slip plane in response to the component $\sigma_{i j} b_{i} m_{j}$ of the applied stress (the so-called Schmid factor [12]), where $\mathbf{b}$ is the Burgers vector and $\mathbf{m}$ is the normal to the slip plane. Thus dislocations in the first family will move in response to $\sigma_{12}$ while those in the second family will move in response to $\sigma_{13}$. It has been experimentally observed (cf. [10]) that the speed of dislocations grows as a power law of the applied stress. Then, in the absence of any interaction between the families we would close the model with velocity laws such as

$$
\begin{aligned}
& v_{1}=\operatorname{sign}\left(\sigma_{12}\right)\left|\sigma_{12}\right|^{\gamma}, \\
& v_{2}=\operatorname{sign}\left(\sigma_{13}\right)\left|\sigma_{13}\right|^{\gamma},
\end{aligned}
$$

where any constant of proportionality may be set equal to unity through a suitable rescaling of time. 


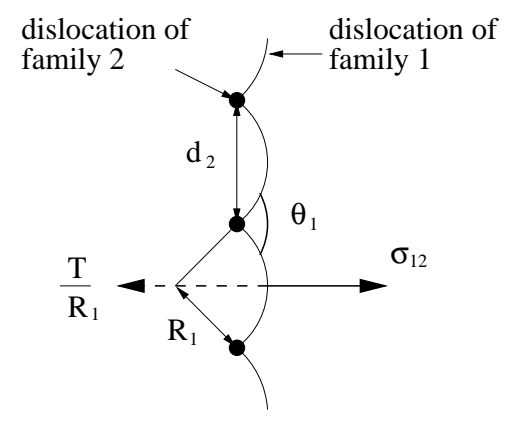

FIG. 2.2. Dislocations of the first family pinned by those of the second family.

In fact, if the applied stress is smaller than a critical threshold (the Peierls stress) then dislocations remain at rest. The speed laws (2.3) are applicable if the applied stresses are large compared with the Peierls stress, which is usually the case, since for many materials the Peierls stress is small.

Note that sheets of edge dislocations generate no stress of their own so that $\sigma_{12}$ and $\sigma_{13}$ here are just the applied external stresses, which are independent of $x$ but may be dependent on time. In our setting, the first family of dislocations can be seen as a set of lines parallel to the $y$-axis, and the second family is another set of lines parallel to the $z$-axis. Both families move along the $x$-axis. However, as dislocations from the first family move they must cut through the dislocations of the second family. We suppose that there is a strong resistance to this cutting. Suppose first that the dislocations in the second family are held fixed. Then for small values of $\sigma_{12}$ the dislocations in the first family will not cut those of the second family, but will form arcs of circles between the pinning dislocations of this family as in Figure 2.2 (such interactions are often known as "forest" interactions).

The classical theory of dislocations establishes the existence of an elastic energy as well as an associated "line tension" associated to each dislocation (cf. [3]). The driving force of the applied stress is then balanced by the curvature induced restoring force, $T / R_{1}$, due to the "line tension," $T$, in a dislocation, where $R_{1}$ is the radius of curvature. Thus, we have

$$
\left|\sigma_{12}\right|=\frac{T}{R_{1}} .
$$

However, there is a minimun value of $R_{1}$ the dislocation can attain while still passing through the pinning sites. For sufficiently large applied stresses neighboring arcs of circles will meet tangentially at the pins, and may reconnect so that the pins have been traversed, as in Figure 2.3.

This happens when the curvature of the dislocation reaches the value $2 / d_{2}$, where $d_{2}$ is the distance between dislocations of the second family. Thus we expect the second family to generate a pinning force on the first family proportional to $\sqrt{w_{2}}$ (since this is proportional to $\left(d_{2}\right)^{-1}$ ). Applying this argument to both families independently we were led in [5] to consider the velocity laws

$$
\begin{aligned}
& v_{1}=\operatorname{sign}\left(\sigma_{12}\right)\left(\left(\left|\sigma_{12}\right|-a \sqrt{w_{2}}\right)^{+}\right)^{\gamma}, \\
& v_{2}=\operatorname{sign}\left(\sigma_{13}\right)\left(\left(\left|\sigma_{13}\right|-a \sqrt{w_{1}}\right)^{+}\right)^{\gamma},
\end{aligned}
$$

where $a=2 T$ may be taken as a parameter. Here and from now on $(s)^{+}=s$ for 


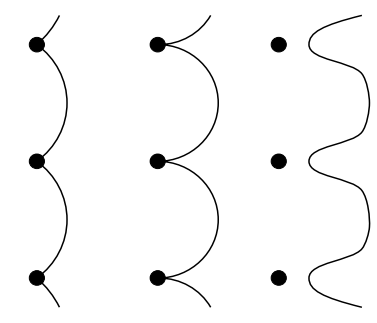

FIG. 2.3. Dislocations of the first family cutting those of the second family.

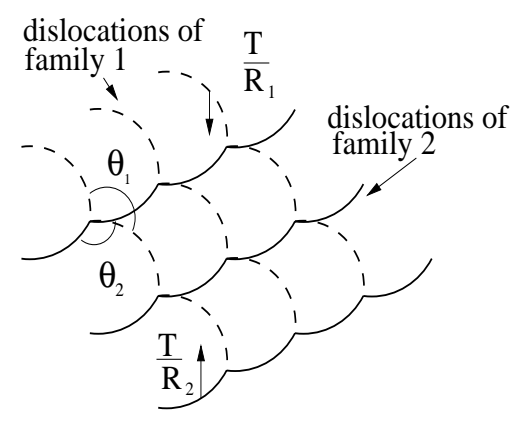

FIG. 2.4. Mutual pinning of dislocations.

$s>0$, and $(s)^{+}=0$ for $s \leq 0$. We will use the speed laws (2.5), (2.6) to close system $(2.1),(2.2)$ in this paper.

Although the resulting model reproduces some interesting dislocation patterns, a more realistic model should assume that both families of dislocations are curved, not just one of them. More precisely, in allowing each family to pin the other we must not fix one family when calculating the pinning effect it has on the other, but allow it to be "dragged along"; we must perform an overall force balance rather than a separate force balance for each family. Thus we are led to consider the situation shown in Figure 2.4, which is a three-dimensional version of Figure 2.2, in which we have represented both families of dislocations and their mutual interaction. Cutting Figure 2.4 across planes perpendicular to one of the families we obtain pictures analogous to Figure 2.2.

Now a local force balance at the crossing points gives that $\theta_{1}=\theta_{2}$ assuming that the line tension in both dislocations is the same, where $\theta_{1}$ is the jump in the angle of the tangent vector of a dislocation of family $i$ at the crossing point (see Figures $2.2,2.4$ ). Hence $d_{2} / R_{1}=d_{1} / R_{2}$. If there is no cutting the dislocations must move with the same velocity. Using (2.4) and the analogous one for the second family of dislocations we obtain

$$
\sigma_{12}-\sigma_{13}=T\left(\frac{1}{R_{1}}+\frac{1}{R_{2}}\right)
$$

We are assuming here that the dislocations deform as in Figure 2.4. This gives

$$
\begin{aligned}
& R_{1}=\frac{T}{\left(\sigma_{12}-\sigma_{13}\right)} \frac{\left(d_{1}+d_{2}\right)}{d_{1}}, \\
& R_{2}=\frac{T}{\left(\sigma_{12}-\sigma_{13}\right)} \frac{\left(d_{1}+d_{2}\right)}{d_{2}} .
\end{aligned}
$$


Inserting these values in (2.4) and in the analogous law for family two, the speed of the dislocations can be expressed as

$$
v_{1}=v_{2}=\operatorname{sign}\left(\frac{d_{1} \sigma_{13}+d_{2} \sigma_{12}}{d_{1}+d_{2}}\right)\left|\frac{d_{1} \sigma_{13}+d_{2} \sigma_{12}}{d_{1}+d_{2}}\right|^{\gamma},
$$

where the dislocation separations are related to the dislocation densities by

$$
w_{1}=\frac{1}{d_{1}^{2}}, \quad w_{2}=\frac{1}{d_{2}^{2}} .
$$

Such a situation can hold so long as there is no cutting, i.e., $R_{1}<\frac{d_{2}}{2}$ and equivalently $R_{2}<\frac{d_{1}}{2}$. Using (2.7)-(2.8)

$$
\left|\sigma_{12}-\sigma_{13}\right|<a\left(\sqrt{w_{1}}+\sqrt{w_{2}}\right),
$$

where $a=2 T$ as before. If this constraint is violated then the dislocations will cut each other, giving $R_{1}=d_{2} / 2, R_{2}=d_{1} / 2$ and

$$
\left.\begin{array}{l}
\left.\begin{array}{l}
v_{1}=\operatorname{sign}\left(\sigma_{12}-a \sqrt{w_{2}}\right)\left|\sigma_{12}-a \sqrt{w_{2}}\right|^{\gamma} \\
v_{2}=\operatorname{sign}\left(\sigma_{13}+a \sqrt{w_{1}}\right)\left|\sigma_{13}+a \sqrt{w_{1}}\right|^{\gamma}
\end{array}\right\} \quad \text { if } \sigma_{12}-\sigma_{13}>a\left(\sqrt{w_{1}}+\sqrt{w_{2}}\right), \\
v_{1}=\operatorname{sign}\left(\sigma_{12}+a \sqrt{w_{2}}\right)\left|\sigma_{12}+a \sqrt{w_{2}}\right|^{\gamma} \\
v_{2}=\operatorname{sign}\left(\sigma_{13}-a \sqrt{w_{1}}\right)\left|\sigma_{13}-a \sqrt{w_{1}}\right|^{\gamma}
\end{array}\right\} \quad \text { if } \sigma_{12}-\sigma_{13}<-a\left(\sqrt{w_{1}}+\sqrt{w_{2}}\right) .
$$

Having considered some general properties of the models (2.5)-(2.6) and (2.9)(2.13) we will simplify the problem by considering a scenario in which one family of dislocations is pinned through some external mechanism, so that only one family of dislocations is free to move. Our aim will be to see how the presence of a (nonuniform) distribution of pinning "trees" will affect the motion of a family of dislocations, and in particular how it may lead to a dislocation pile-up.

We will find that the simple model we have formulated may be ill-posed in some regions of parameter space and may develop singularities. This is because, as mentioned earlier, pure sheets of edge dislocations generate no stress to leading order, so that there is no repulsion mechanism preventing infinite dislocation densities. Because of this, we will also study a simple regularized model in which we include a small force on the dislocations proportional to the gradient of the density, which will model the higher order repulsion effects of sheets of edges and will smooth out the singularities present in the original model. Thus we will set

$$
\frac{\partial w_{1}}{\partial t}+\frac{\partial}{\partial x}\left(w_{1}\left(\left(\sigma_{12}-a \sqrt{w_{2}}-\epsilon \frac{\partial w_{1}}{\partial x}\right)^{+}\right)^{\gamma_{1}}\right)=0
$$

where we have assumed without loss of generality that $\sigma_{12}$ is positive.

Our regularization here is rather "ad hoc"; we have made this choice because it is a simple mathematical law that yields a local model and reproduces the expected self-repulsion between dislocations of the same type. It would be nice to calculate the higher order correction terms when passing from a discrete dislocation model to the dislocation density model (which may be nonlocal), and to check if they can be approximated in some limit by the second order terms in (2.14). 


\section{Pile-up solutions for the system of conservation laws.}

3.1. Remarks on stability and well-posedness. From now on, we assume that the applied stresses are independent of time. We are concerned here with finding solvability conditions for (2.1)-(2.2) with either (2.5)-(2.6) or (2.9)-(2.13).

First of all let us check whether the system is hyperbolic by computing the Jacobian of the flux. With $U=\left(w_{1}, w_{2}\right)^{t}$ we have

$$
U_{t}+(F(U))_{x}=U_{t}+D F(U) U_{x}=0,
$$

where $F(U)=\left(w_{1} v_{1}\left(w_{2}\right), w_{2} v_{2}\left(w_{1}\right)\right)^{t}$ so that

$$
D F(U)=\left(\begin{array}{cc}
v_{1}\left(w_{2}\right) & \frac{\partial v_{1}\left(w_{2}\right)}{\partial w_{2}} w_{1} \\
\frac{\partial v_{2}\left(w_{1}\right)}{\partial w_{1}} w_{2} & v_{2}\left(w_{1}\right)
\end{array}\right) .
$$

The system (2.1), (2.2) is said to be strictly hyperbolic if $D F(U)$ has two real distinct eigenvalues $\lambda_{1}, \lambda_{2}$. If the eigenvalues are real and equal, the system is said to be nonstrictly hyperbolic. In both cases, the characteristic curves (solutions to $\lambda^{\prime}(s)=$ $\left.\lambda_{i}\right)$ are real. When the eigenvalues are complex, the characteristics are complex and the system is said to be elliptic. The discriminant for the equation whose roots are the eigenvalues is given by

$$
\Delta\left(w_{1}, w_{2}\right)=\left(v_{1}\left(w_{2}\right)-v_{2}\left(w_{1}\right)\right)^{2}+4 w_{1} w_{2} \frac{\partial v_{2}\left(w_{1}\right)}{\partial w_{1}} \frac{\partial v_{1}\left(w_{2}\right)}{\partial w_{2}} .
$$

For either $(2.5)-(2.6)$ or $(2.9)-(2.13), \Delta\left(w_{1}, w_{2}\right)$ may be positive or negative in different regions of the $\left(w_{1}, w_{2}\right)$ plane. Therefore, our system is of mixed type. Figures 3.1 and 3.2 show the change of type in the system when $\gamma_{i}=1$ for (2.5)-(2.6) and (2.9)-(2.13), respectively. Notice that the elliptic region for (2.5)-(2.6) appears only when the speeds of both families of dislocations have opposite sense. Let us also point out that in the nonstrictly hyperbolic region this model reduces to the trivial system $\frac{\partial w_{1}}{\partial t}=0=\frac{\partial w_{2}}{\partial t}$. This is due to the existence of threshold values for the stresses below which the speeds are zero.

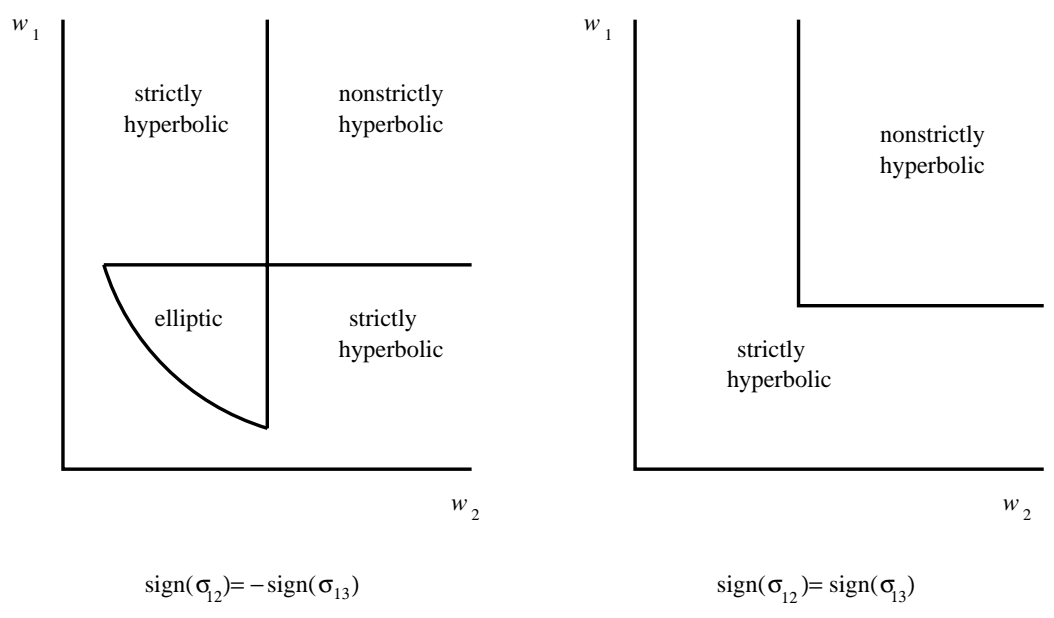

FIG. 3.1. Change of type in the model (2.5)-(2.6). 


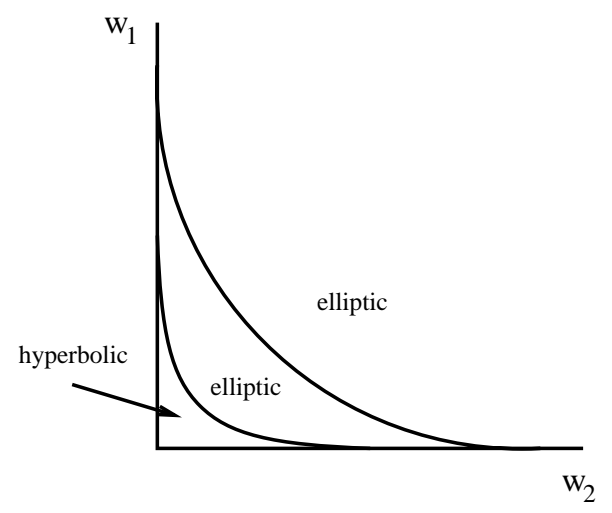

FIG. 3.2. Change of type in the model (2.9)-(2.13).

Since our problem is time dependent, the appearance of an elliptic region results in ill-posedness of the initial value problem (2.1), (2.2). Let us examine the linear stability of a particular class of solutions, namely the constant solutions. Linearizing about the constant state $\left(\bar{w}_{1}, \bar{w}_{2}\right)$ by setting $\left(w_{1}, w_{2}\right)=\left(\bar{w}_{1}+u_{1}, \bar{w}_{2}+u_{2}\right)$, we find

$$
\begin{aligned}
& u_{1, t}+v_{1}\left(\bar{w}_{2}\right) u_{1, x}+\bar{w}_{1} \frac{\partial v_{1}}{\partial w_{2}}\left(\bar{w}_{2}\right) u_{2, x}=0, \\
& u_{2, t}+v_{2}\left(\bar{w}_{1}\right) u_{2, x}+\bar{w}_{2} \frac{\partial v_{2}}{\partial w_{1}}\left(\bar{w}_{1}\right) u_{1, x}=0 .
\end{aligned}
$$

This system has plane wave solutions of the form

$$
\left(u_{1}, u_{2}\right)=\left(l_{1}, l_{2}\right) e^{i(\omega t+k x)}
$$

with the dispersion law $\omega=\omega(k)$ given by

$$
\omega(k)=k\left(\frac{-\operatorname{tr}(A) \pm \sqrt{\operatorname{tr}(A)^{2}-4 \operatorname{det}(A)}}{2}\right),
$$

where

$$
A=\left(\begin{array}{cc}
v_{1}\left(\bar{w}_{2}\right) & \bar{w}_{1} \frac{\partial v_{1}}{\partial w_{2}}\left(\bar{w}_{2}\right) \\
\bar{w}_{2} \frac{\partial v_{2}}{\partial w_{1}}\left(\bar{w}_{1}\right) & v_{2}\left(\bar{w}_{1}\right)
\end{array}\right) .
$$

We see that $\omega(k) / k$ is an eigenvalue of $A$ with eigenvector $l=\left(l_{1}, l_{2}\right)$. When $A$ has complex eigenvalues, that is, when $\Delta\left(\bar{w}_{1}, \bar{w}_{2}\right)=\operatorname{tr}(A)^{2}-4 \operatorname{det}(A)<0$, we find solutions with unbounded exponential growth for high frequencies, so that the system is linearly unstable with unbounded growth rate in the elliptic region.

The most interesting interactions occur away from the strictly hyperbolic regime. This fact has also been observed in other models, for instance, in [16] the change in type from hyperbolic to elliptic marks the onset of shear band formation.

In this paper we will restrict ourselves to the analysis of model (2.5)-(2.6) and moreover, we will assume that one of the dislocation densities is large enough to pin the other. Under this assumption, the system, that reduces to an scalar equation, remains in the hyperbolic (either strictly or nonstrictly) region. We show in section 3.2 an example of a solution of (2.5)-(2.6) entering the nonstrictly hyperbolic region, which describes the pile-up of a family of dislocations. 
As usual in hyperbolic systems nonuniqueness problems arise due to the presence of discontinuities. For this reason we have also analyzed the regularized model (2.14). Assuming that (2.14) has indeed a physical sense, the only reasonable solutions for $(2.5)-(2.6)$ are those which can be obtained as limits of (2.14) as $\epsilon$ tends to zero. From the mathematical point of view, this is analogous to the regularization of scalar hyperbolic equations by using viscosity terms. However, there are some technical differences. Due to the degeneracy of (2.14), the regularized problem in our case is a free-boundary problem with viscosity acting only on one side of the free boundary.

3.2. Elementary pile-up solutions. We next construct an elementary example of a solution where pile-up occurs. As described in section 2, we will restrict our attention to situations where the second family is held fixed through some exterior mechanism, and examine its influence on the motion of the first family. We will assume that the density of the second family is large enough to pin the first family in some regions of space, but not in others, where the evolution of the density can be readily computed by the method of characteristics. We assume first that the stress field is such as to move these dislocations toward the region of the space where they are pinned, resulting in a pile-up phenomenon.

We start from an initial smooth density $w_{1,0}(x)$ and take $w_{2}(x)$ to be smooth and monotonically increasing from $\alpha$ to $\beta$ with $0<\alpha<k=\sigma_{12}^{2} / a^{2}=w_{2}(0)<\beta$. Then

$$
w_{1, t}+\left(\left(\left(\sigma_{12}-a \sqrt{w_{2}(x)}\right)^{+}\right)^{\gamma} w_{1}\right)_{x}=0 .
$$

Let $c(x)=\left(\left(\sigma_{12}-a \sqrt{w_{2,0}(x)}\right)^{+}\right)^{\gamma}$. By our choice of $w_{2}$, we see that, for $x>0$ the coefficient $c(x)$ vanishes and $w_{1}(x, t)=w_{1,0}(x)$.

When $x<0$, the evolution of $w_{1}$ is given by (3.5). Set $v(x, t)=w_{1}(x, t) c(x)$. Then

$$
v_{t}+c(x) v_{x}=0
$$

Thus, $v(x, t)$ is constant along the characteristic curves $x(t)$, which are the solutions of

$$
\frac{d x(t)}{d t}=c(x(t))=\left(\left(\sigma_{12}-a \sqrt{w_{2}(x(t))}\right)^{+}\right)^{\gamma} \geq 0 .
$$

This yields the formula

$$
w_{1}(x(t), t)=w_{1,0}(x(0)) \frac{c(x(0))}{c(x(t))} \geq w_{1,0}(x(0))>k_{2}
$$

when $c(x(t)) \neq 0$, so that $w_{1}$ is increasing. Generically, we can assume $\frac{\partial w_{2}(0)}{\partial x}>0$, so that $\sigma_{12}-a \sqrt{w_{2}(x)} \sim-\lambda x$ with $\lambda>0$ as $x \rightarrow 0^{-}$.

Then, it follows from (3.7) that, for $x$ small and negative, the equation for the characteristics may be approximated by

$$
\frac{d x}{d t}(t) \approx(-\lambda x(t))^{\gamma}
$$

We must distinguish three cases.

Case 1. $\gamma>$ 1. Integrating (3.9), we obtain for small $x(0)<0$

$$
x(t) \sim-\frac{1}{\left(\lambda^{\gamma}(\gamma-1) t+(-x(0))^{1-\gamma}\right)^{\frac{1}{\gamma-1}}}<0 .
$$


Notice that, in this case, the characteristics starting at negative values remain negative for all times. Also, $x(t) \rightarrow 0$ as $t \rightarrow \infty$.

Then,

$$
x(0) \sim \frac{x}{\left(1-\lambda^{\gamma}(\gamma-1) t(-x)^{\gamma-1}\right)^{\frac{1}{\gamma-1}}} .
$$

Using (3.8) and (3.11) we deduce

$$
w_{1}(x, t)=w_{1,0}(x(0)) \frac{c(x(0))}{c(x)} \rightarrow w_{1,0}(0)=w_{1}\left(0^{+}, t\right)
$$

as $x \rightarrow 0^{-}$. Notice that the function $w_{1}(x, t)$ is continuous at $x=0$.

Case 2. $\gamma=1$. Integrating (3.9), it follows that for small $x(0)<0$ the characteristics are given by

$$
x(t)=x(0) e^{-\lambda t}
$$

which again remain negative at all times. Notice that $x(t)$ tends to 0 as $t \rightarrow \infty$. Using (3.8) and (3.13) we conclude

$$
w_{1}(x, t) \approx w_{1,0}\left(x e^{\lambda t}\right) e^{\lambda t} .
$$

Letting $x \rightarrow 0^{-}$for each $t$ fixed, we get

$$
w_{1}\left(0^{-}, t\right)=e^{\lambda t} w_{1,0}(0)=e^{\lambda t} w_{1}\left(0^{+}, t\right)
$$

so that $w_{1}(x, t)$ jumps at $x=0$ for every $t>0$. We construct a global solution for $x \in R$ to (3.5) by the method of characteristics defining $w_{1}(x, t)=w_{1,0}(x)$ for $x>0$ and as the solution of (3.5) given by (3.8) for $x<0$. By standard arguments, $w_{1}$ defined in this way is a weak solution if the following Rankine-Hugoniot condition is satisfied:

$$
0=\frac{c\left(0^{-}\right) w_{1}\left(0^{-}, t\right)-c\left(0^{+}\right) w_{1}\left(0^{+}, t\right)}{w_{1}\left(0^{-}, t\right)-w_{1}\left(0^{+}, t\right)} .
$$

This identity is a consequence of (3.5) and the definition of $w_{1}$ for $x>0$.

Case 3. $0<\gamma<1$. In this case, the solution of (3.9) is given by

$$
x(t)=-\left((-x(0))^{1-\gamma}-\lambda^{\gamma}(1-\gamma) t\right)^{\frac{1}{1-\gamma}}
$$

for $x(0)<0$ small. Notice that, for $T_{c}=\frac{(-x(0))^{1-\gamma}}{\lambda^{\gamma}(1-\gamma)}$, we obtain $x\left(T_{c}\right)=0$.

Using (3.8), (3.17), and letting $x \rightarrow 0^{-}$, we get

$$
w_{1}(x, t) \sim w_{1,0}\left(-\left(\lambda^{\gamma}(1-\gamma) t\right)^{\frac{1}{1-\gamma}}\right) \frac{c\left(-\left(\lambda^{\gamma}(1-\gamma) t\right)^{\frac{1}{1-\gamma}}\right)}{\lambda^{\gamma}|x|^{\gamma}} .
$$

We see that $w_{1}(x, t)$ blows up as $x \rightarrow 0^{-}$for every $t>0$.

Notice that along a characteristic $x(t)$ starting at $x(0)<0, w_{2}(x(t))$ tends to $\frac{\sigma_{12}^{2}}{a^{2}}$. Thus, the density $w_{1}(x(t), t)$ tends to infinity as $t \rightarrow \infty$ while it moves with a speed $c(x(t))$ decreasing to zero. This shows that $w_{1}$ piles-up at $x=0$ for large times. We illustrate schematicaly this phenomenon in Figures 3.3 and 3.4. 

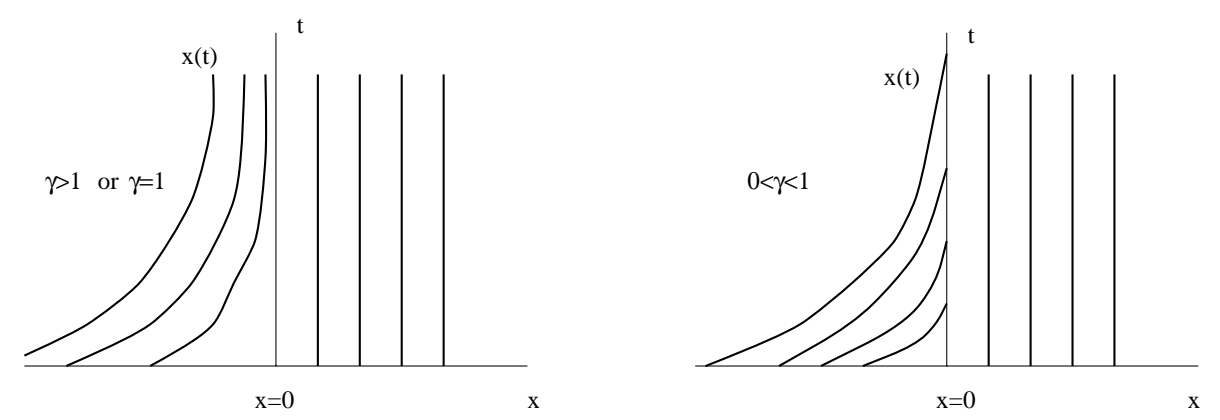

FIG. 3.3. Characteristics.
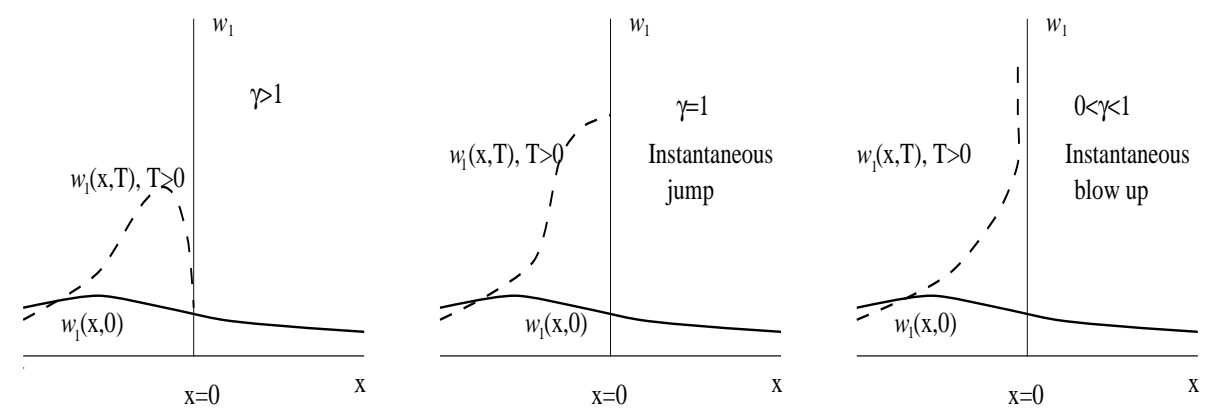

FIG. 3.4. Profiles of $w_{1}$ for $t=0$ and $t=T>0$. We illustrate the changes for $x<0$, depending on $\gamma$.

Summarizing, the analysis above shows in all three cases the function $w_{1}(x, t)$ piles up near $x=0$. However, the detailed way in which this happens depends on $\gamma$. For $\gamma>1, w_{1}(x, t)$ remains continuous. For $\gamma=1$, it develops a jump discontinuity at $x=0$. The amplitude of the jump increases exponentially with time. We will see later that this jump is smoothed out if we solve (2.14) instead of (2.1), (2.5), keeping the same initial data. Otherwise, if $0<\gamma<1, w_{1}(x, t)$ blows up at $x=0$ for every $t>0$.

Remark. The solutions we have constructed for $\gamma \geq 1$ are weak solutions in the sense that they satisfy the integral identity

$$
\int_{0}^{\infty} \int_{\mathbb{R}}\left(w_{1} \phi_{t}+c w_{1} \phi_{x}\right) d x d t=\int_{\mathbb{R}} w_{1}(x, 0) \phi(x, 0) d x
$$

for all $\phi \in C_{c}(\mathbb{R} \times[0, \infty))$. For $\gamma>1$ they are classical and unique by standard results (cf. [15]).

3.3. Formation of regions of low density of dislocations. Here we consider the case in which the stress field is such as to move the dislocations away from the pinned region. In between the pinned region and the retreating dislocations a region of low dislocation density should be created. We assume a smooth initial density $w_{1,0}(x)$ and take $w_{2}(x)$ to be continuous, monotonically decreasing from $\alpha$ to $\beta$ with $\alpha>k=w_{2}(0)>\beta$ and $k=\sigma_{12}^{2} / a^{2}$ as before. To determine $w_{1}$ for $x>0$, we study the solution of (3.5). It is given by formula (3.8) with $x(t)$ satisfying (3.7) such that $x(t)=x$. Now, for $x(0)>0, x(s)$ increases and $c(x(t))>0$. When $\gamma \geq 1$, 

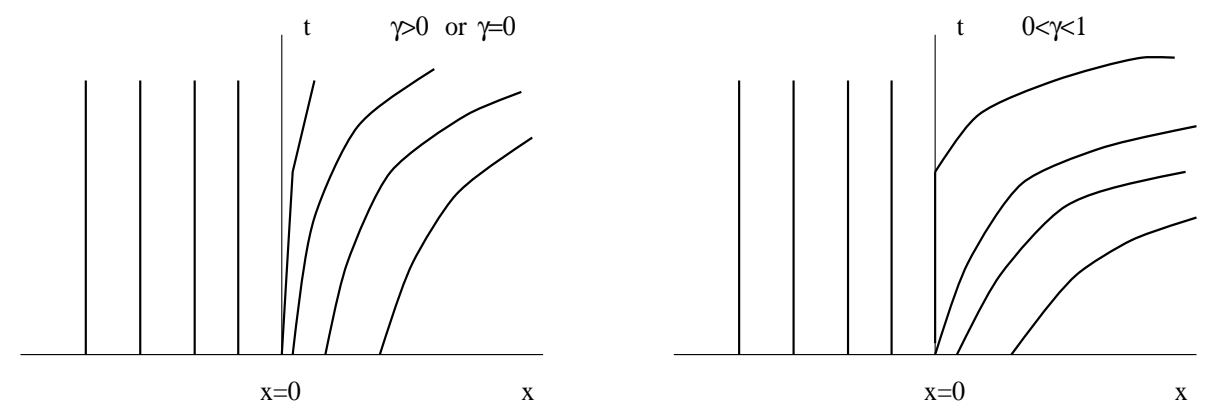

FIG. 3.5. Characteristics.
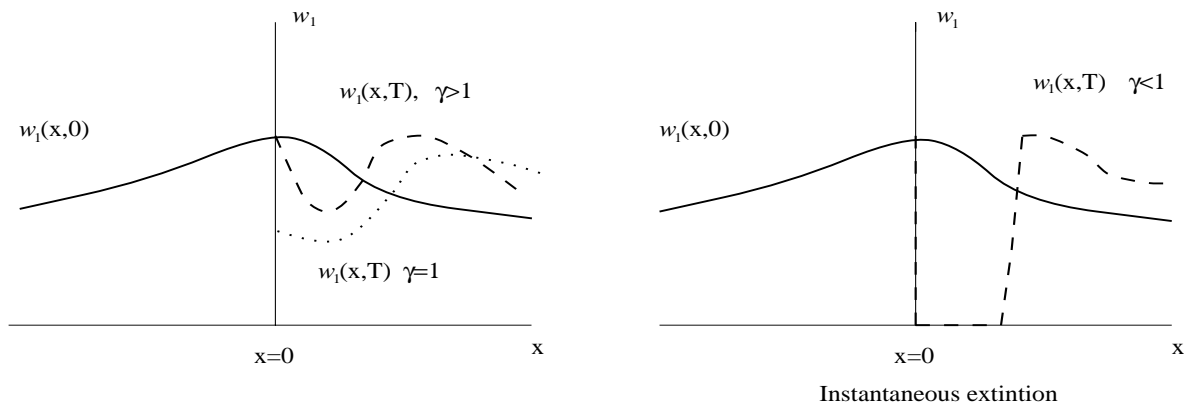

FIG. 3.6. Profiles of $w_{1}$ for $t=0$ and $T>0$. We illustrate the changes in the profiles for $x>0$, depending on $\gamma$.

the characteristic curves $x(t), s \geq 0$ with $x(0)>0$ cover the region $x>0, t>0$ and the solution is fully determined. We observe now that $w_{1}(x(t), t)$ decreases with time. We illustrate this phenomenon schematically in Figures 3.5 and 3.6. Near zero, $c(x) \approx(\lambda x)^{\gamma}$. A local analysis shows that for $\gamma>1$ the solution is continuous. If $\gamma=1$ a jump appears.

When $0<\gamma<1, w_{1}$ takes the value zero in the region $(x, t), 0<x<x_{0}(t)$, where $x_{0}(t)$ is the characteristic starting from $x_{0}(0)=0$. We have instantaneous extinction of the solutions.

4. Regularized solutions. In this section we study the well-posedness of the regularized model (2.14) in a rigorous way. As a consequence of this analysis we will also obtain that the solution behaves in a self-similar manner for small times.

4.1. Formulation of the problem. Motivated by experiments and numerical simulations (see [3], [10]) which indicate that $\gamma$ is close to one we restrict ourselves to the particular case $\gamma=1$, which is simpler mathematically.

Our goal is to analyze how the regularizing terms smooth out the discontinuities that appear in solutions to (3.5) when $\gamma=1$ and $\epsilon$ approaches to zero. We choose the same initial data for $w_{1}$ and a similar fixed $w_{2}(x)$.

We can construct solutions of (2.14) by solving an auxiliary-free-boundary problem. With $c(x)=\sigma_{12}-a \sqrt{w_{2}(x)}$ as before problem (2.14) is

$$
w_{1, t}+\left(\left(c(x)-\epsilon w_{1, x}\right)^{+} w_{1}\right)_{x}=0
$$




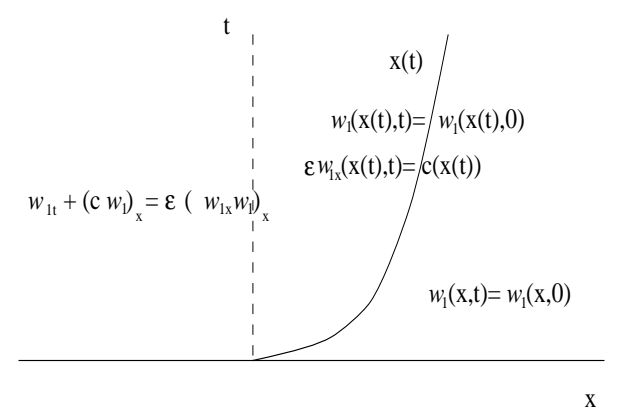

FIG. 4.1. Structure of $w_{1}$.

with initial data

$$
w_{1}(x, 0)=w_{1,0}(x)
$$

We will construct particular solutions of (4.1), (4.2) where $c(x)-\epsilon w_{1, x}$ changes sign once at $x=\lambda(t)$ (see Figure 4.1). More precisely, in the region $x<\lambda(t)$,

$$
w_{1, t}+\left(c(x) w_{1}\right)_{x}=\epsilon\left(w_{1, x} w_{1}\right)_{x},
$$

while, for $x>\lambda(t)$,

$$
w_{1, t}(x, t)=0
$$

On the curve $x=\lambda(t)$ we have

$$
c(\lambda(t))=\epsilon w_{1, x}(\lambda(t), t) .
$$

Moreover, it is reasonable on physical grounds to assume that $w_{1}$ is continuous across $\lambda(t)$, namely,

$$
w_{1}\left(\lambda(t)^{-}, t\right)=w_{1}\left(\lambda(t)^{+}, t\right) .
$$

It is easily checked that every solution of the free-boundary problem (4.2)-(4.6) solves (4.1), (4.2) in the sense of distributions only if the additional condition

$$
(x-\lambda(t))\left(c(x)-\epsilon w_{1, x}\right)<0, x \neq \lambda(t)
$$

is satisfied. By a solution in the sense of distributions we mean

$$
\int_{0}^{\infty} \int_{\mathbb{R}}\left(w_{1} \phi_{t}+\left(c(x)-\epsilon w_{1, x}\right)^{+} w_{1} \phi_{x}\right) d x d t=\int_{\mathbb{R}} w_{1,0}(x) \phi(x, 0) d x
$$

for all $\phi \in C_{c}(\mathbb{R} \times[0, \infty))$.

4.2. Well-posedness and uniqueness. Some comments on the well-posedness of the initial value problem for (4.1) are in order. We will consider later the existence of solutions. The uniqueness of (4.1), (4.2) is a nonclassical problem due to the nonuniform parabolicity of the equation. We will not address this problem in this paper in detail. However, it is interesting to observe that we can trivially construct an infinite family of solutions to the free-boundary problem (4.2)-(4.6) (but in principle 
not of the parabolic equations (4.1), (4.2)) taking any arbitrary smooth decreasing function $\lambda(t)$. To do so, we solve the boundary value problem (4.2), (4.3), (4.5) for any $\lambda(t)$ decreasing fixed. This yields a value for $w_{1}\left(\lambda^{-}(t), t\right)$ so that we can define $w_{1}(x, t)=w_{1}\left(\lambda^{-}(t), t\right)$ by means of (4.6) for $\lambda(t) \leq x \leq \lambda(0)$. When $x>\lambda(0)$, $w_{1}(x, t)=w_{1,0}(x)$.

Nevertheless, the solutions constructed in this way are not solutions of (4.1), (4.2) since they should also satisfy the extra condition (4.7). It turns out that these extra conditions do not hold. Indeed, differentiating the boundary condition (4.6) with respect to $t$ and using (4.4) we get $w_{x}^{-} \lambda^{\prime}+w_{t}=w_{x}^{+} \lambda^{\prime}$. Using (4.3) we may rewrite this as

$0<\left(w_{x}\left(\lambda(t)^{+}, t\right)-c(\lambda(t))\right)-\left(w_{x}\left(\lambda(t)^{-}, t\right)-c(\lambda(t))\right)=\left(-c+w_{x}\right) w_{x}+\left(-c^{\prime}+w_{x x}\right) w$.

Taking into account that for $x=\lambda(t), c(x)+w_{x}$ vanishes, using (4.7) as well as $\lambda^{\prime}(t)<0$, we conclude that $w_{x x}<c^{\prime}(x)$. Let $u=w_{x}-c$. Then

$$
\begin{array}{r}
u=0, \quad u_{x}<0 \text { at } x=\lambda(t), \\
u<0, \quad x<\lambda(t) .
\end{array}
$$

Now, $u$ solves the parabolic equation $u_{t}=\left(w_{x} u+w u_{x}\right)_{x}$. Taking into account (4.8), (4.9) we obtain a contradiction.

Summarizing, the solutions of the free-boundary problem (4.2)-(4.6) with $\lambda^{\prime}(t)<$ 0 are not solutions of (4.1), (4.2). We will see later that it is possible to construct solutions of the free-boundary problem with $\lambda^{\prime}(t)>0$ satisfying (4.7) and therefore solving (4.1), (4.2).

4.3. Reformulation of the free-boundary problem. We now rewrite the problem in a more convenient way for the analysis of well-posedness. Let us define $z(x, t)=\frac{\partial w_{1}}{\partial x}$. Differentiating (4.3) with respect to $x$ we obtain for $x<\lambda(t), t>0$

$$
\frac{\partial z}{\partial t}+\left(c^{\prime \prime}(x)-\epsilon \frac{\partial^{2} z}{\partial x^{2}}\right) w_{1}+2\left(c^{\prime}(x)-\epsilon \frac{\partial z}{\partial x}\right) z+(c(x)-\epsilon z) \frac{\partial z}{\partial x}=0 .
$$

If we assume $\lambda^{\prime}>0$, then $w_{1}(x, t)=w_{1,0}(x)$ for $x>\lambda(t)$ and (cf. (4.4))

$$
w_{1}(\lambda(t), t)=w_{1,0}(\lambda(t)) .
$$

Differentiating this identity we obtain

$$
\left(\frac{\partial w_{1,0}}{\partial x}(\lambda(t))-\frac{1}{\epsilon} c(\lambda(t))\right) \lambda^{\prime}(t)=\left(c^{\prime}(\lambda(t))-\epsilon \frac{\partial z}{\partial x}(\lambda(t), t)\right) w_{1,0}(\lambda(t)) .
$$

The free-boundary condition (4.5) reads

$$
c(\lambda(t))=\epsilon z(\lambda(t), t) .
$$

Problem (4.10), (4.12), (4.13) now has a resemblance with the classical Stefan problem, which has been widely studied and for which a large wealth of mathematical techniques are available. In order to further simplify the problem, we introduce a new variable $u(x, t)=\epsilon z(x, t)-c(x)$.

In this new variable, problem (4.10), (4.12), (4.13) becomes

$$
\begin{array}{r}
u_{t}-\epsilon w_{1} u_{x x}-2(u+c) u_{x}-u\left(u_{x}+c^{\prime}\right)=0, \quad x<\lambda(t), \\
u(\lambda(t), t)=0, \\
u(\lambda(t), 0) \lambda^{\prime}(t)=\epsilon w_{1,0}(\lambda(t)) \frac{\partial u(\lambda(t), t)}{\partial x} .
\end{array}
$$


Problem (4.14)-(4.16) has several analogies to the Stefan problem. However, a technical difficulty arises at time zero. The starting point for the free boundary $\lambda(t)$ is characterized by means of

$$
c(\lambda(0))=\epsilon \frac{\partial w_{1,0}(\lambda(0))}{\partial x}
$$

or, in an equivalent way, $u(\lambda(0), 0)=0$. In particular, this implies that condition (4.16) becomes singular at time $t=0$. In order to understand better where the difficulty lies, we approximate (4.14)-(4.16) by the leading order terms near $x=\lambda(0)$. By convenience, we make the following assumption that holds for quite general initial data:

$$
\frac{\partial u}{\partial x}(\lambda(0), 0)=\Gamma \neq 0 .
$$

This implies $u(x, 0) \sim \Gamma(x-\lambda(0))$ as $x \rightarrow \lambda(0)$.

Keeping the leading order terms of (4.14)-(4.16) in a neighborhood of $x=\lambda(0)$ we end up with the following problem:

$$
\begin{array}{r}
\frac{\partial u}{\partial t}=\epsilon w_{1}(\lambda(0)) \frac{\partial^{2} u}{\partial^{2} x}, \quad x<\lambda(t), \\
u(\lambda(t), t)=0, \\
\Gamma(\lambda(t)-\lambda(0)) \lambda^{\prime}=\epsilon w_{1}(\lambda(0)) \frac{\partial u}{\partial x}(\lambda(t), t)
\end{array}
$$

complemented with the initial condition $u(x, 0)=\Gamma(x-\lambda(0))$.

Problem (4.19)-(4.21) admits a self-similar solution with increasing $\lambda(t)$ in the form

$$
\begin{array}{r}
\hat{u}(x, t)=\sqrt{\epsilon w_{1}(0) t} \phi\left(\frac{x-\lambda(0)}{\sqrt{\epsilon w_{1}(0) t}}\right), \\
\hat{\lambda}(t)=\lambda(0)+\xi_{0} \sqrt{\frac{\epsilon w_{1}(0) t}{|\Gamma|}}, \\
y=\frac{x-\lambda(0)}{\sqrt{\epsilon w_{1}(0) t}} .
\end{array}
$$

The detailed construction of this solution is given in Appendix A. In the next section we discuss how to construct solutions of (4.14)-(4.16) as perturbations of this self-similar solution as $t \rightarrow 0$.

4.4. Perturbations of the self-similar solution. We want to obtain solutions of the original problem as perturbations of the self-similar solution to the leading order approximation as $t \rightarrow 0$. Throughout this section we keep $\epsilon$ fixed. Then, we will ignore the dependence on $\epsilon$ of all the constants appearing here. We will consider the behavior as $\epsilon \rightarrow 0$ in the next section. 
We assume that the initial data are smooth enough. More precisely, we assume that

$$
u(x, 0)=\Gamma(x-\lambda(0))+O\left((x-\lambda(0))^{2}\right)<0, \quad x \rightarrow \lambda(0) .
$$

We also assume $w_{1,0}(x)$ to be globally bounded:

$$
0<\frac{1}{K}<w_{1,0}(x)<K
$$

The main result of this section is the following.

THEOREM 4.1. For any initial datum $w_{1,0}(x)$ satisfying (4.25), (4.26), smooth enough, and for any bounded $c \in C^{2}$, there exists a global solution of the free-boundary problem (4.2)-(4.6) where $\lambda(t) \in C^{1+\alpha}(0, \infty), 0<\alpha<\frac{1}{2}$. Moreover, $\lambda(t)=\xi_{0} \sqrt{t}(1+$ $o(1))$ as $t \rightarrow 0^{+}$.

Remark. Near $t=0$ the asymptotic behavior of $w_{1}(x, t)$ can be obtained by means of the self-similar solution (4.22), (4.23) discussed in Appendix A.

Sketch of the proof. The main difficulty in the proof of Theorem 4.1 is the fact that the free boundary for the self-similar solution (4.22), (4.23) behaves as a square root as $t \rightarrow 0^{+}$. Actually, the same will happen for the solutions of the whole freeboundary problem (4.2)-(4.6). Without this difficulty the solution could be a more or less standard adaptation of the fixed point argument used for the analysis of the classical one-dimensional Stefan problem (cf. [9]).

In order to sort out this difficulty we will approximate (4.2)-(4.6) by means of

$$
\begin{array}{r}
\frac{\partial w_{1, n}}{\partial t}+\frac{\partial}{\partial x}\left(\left(c(x)-\epsilon \frac{\partial w_{1, n}}{\partial x}\right) w_{1, n}\right)=0, \quad x<\lambda_{n}(t), t>t_{n}, \\
c\left(\lambda_{n}(t)\right)=\epsilon \frac{\partial w_{1, n}}{\partial x}(\lambda(t), t), \\
w_{1, n}\left(\lambda_{n}(t), t\right)=w_{1,0}\left(\lambda_{n}(t)\right), \\
w_{1, n}\left(x, t_{n}\right)=\Omega_{0}\left(x, t_{n}\right), \quad x<\lambda_{n}\left(t_{n}\right),
\end{array}
$$

with $t_{n}=\frac{1}{n}, \lambda_{n}\left(t_{n}\right)=\hat{\lambda}\left(t_{n}\right)=\xi_{0} \sqrt{\frac{1}{n}}+\lambda(0)$. Notice that the free boundaries $\lambda_{n}(t)$ start at time $t_{n}$ at the free boundary of the self-similar solution. Also, we take as initial data for $w_{1, n}$ at time $t_{n}$ an approximation $\Omega_{0}\left(x, t_{n}\right)$ that we obtain by integrating $u(x, t)=\epsilon \frac{\partial w_{1,0}}{\partial x}(x, t)-c(x)$ with the self-similar solution given in (A.1), (4.23) evaluated at $t_{n}$ as initial data.

Now, since $\lambda_{n}\left(t_{n}\right) \neq 0$, problem (4.27)-(4.30) can be solved by means of a slight adaptation of the classical techniques for the Stefan problem (cf. [8], [9]). However, the time of existence for the solutions, as well as the estimates derived for such a problem could be strongly dependent on $n$. In the technical analysis that will be made in Appendix B, we will show that this is not the case. More precisely, we will prove there that there exists a $\delta>0$ independent on $n$ such that (B.10)-(B.12) hold, where $C$ is independent of $n$.

Taking the limit $n \rightarrow \infty$, we will obtain a solution of (4.2)-(4.6) in a time interval $[0, \delta)$. As a final step, we will obtain a global existence result by means of suitable sub- and supersolutions, as usual in the classical one-dimensional Stefan problem. 
5. Asymptotics of the solutions as $\boldsymbol{\epsilon} \rightarrow \mathbf{0}$. In this section, we describe formally the asymptotics of the free-boundary problem (4.2)-(4.6) as $\epsilon \rightarrow 0$. We recall that such a free-boundary problem can be rewritten as

$$
\begin{array}{rc}
u_{t}-\epsilon w_{1} \frac{\partial^{2} u}{\partial x^{2}}-2(u+c) \frac{\partial u}{\partial x}-u\left(\frac{\partial u}{\partial x}+c^{\prime}\right)=0, & x<\lambda(t), \\
u(x, t)=0, & x=\lambda(t), \\
(\Gamma(x-\lambda(0))+\nu(x)) \dot{x}=\epsilon w_{1,0}(x) \frac{\partial u}{\partial x}(x, t), & x=\lambda(t), \\
\frac{\partial w_{1}}{\partial x}=\frac{1}{\epsilon}(c(x)+u), & x<\lambda(t), \\
w_{1}(x, t)=w_{1,0}(x), & x=\lambda(t), \\
u(x, 0)=\Gamma(x-\lambda(0))+\nu(x), \nu(x)=O\left((x-\lambda(0))^{2}\right), \\
c(x) \sim-k x, x \rightarrow 0, k>0,
\end{array}
$$

where the starting point $\lambda(0)$ is given by

$$
\epsilon \frac{\partial w_{1,0}}{\partial x}(\lambda(0))=c(\lambda(0))
$$

Taking into account $(5.7),(5.8)$ and assuming that $w_{1,0}$ smooth enough, we readily obtain the following asymptotics as $\epsilon$ tends to zero:

$$
\begin{array}{r}
\lambda(0) \sim-\frac{\epsilon}{k} \frac{\partial w_{1,0}}{\partial x}(0)+O\left(\epsilon^{2}\right), \\
\Gamma \sim k+O(\epsilon) .
\end{array}
$$

It then follows that

$$
\Gamma \sim k+\epsilon\left(\frac{\partial^{2} w_{1,0}}{\partial x^{2}}(0)+\frac{c^{\prime \prime}(0)}{k} \frac{\partial w_{1,0}}{\partial x}(0)\right)+o(\epsilon) .
$$

To study the region close to the origin we introduce the change of variables

$$
\begin{array}{r}
x=\lambda(0)+\sqrt{\epsilon} \xi, \\
u=\sqrt{\epsilon} U, \\
\lambda(t)=\lambda(0)+\sqrt{\epsilon} X(t)
\end{array}
$$

that transforms (5.1)-(5.7) into

$$
\begin{aligned}
& U_{t}-w_{1} U_{\xi \xi}-2(\sqrt{\epsilon} U+c) \frac{1}{\sqrt{\epsilon}} U_{\xi}-U\left(U_{\xi}+c^{\prime}\right)=0, \quad \xi<X(t), \\
& U(X(t), t)=0, \\
& \left(\Gamma \xi+\frac{1}{\sqrt{\epsilon}} \nu(\lambda(0)+\sqrt{\epsilon} \xi)\right) \dot{X}(t)=w_{1,0} U_{\xi}, \quad \xi=X(t), \\
& w_{1, \xi}=\frac{1}{\sqrt{\epsilon}}(c+\sqrt{\epsilon} U), \quad \xi<X(t), \\
& w_{1}(X(t), t)=w_{1,0}(X(t)), \\
& u(\xi, 0)=\Gamma \xi+o\left(\sqrt{\epsilon} \xi^{2}\right) .
\end{aligned}
$$


Taking into account $(5.7),(5.10)$, we reduce, to leading order, the problem above to

$$
\begin{array}{r}
U_{t}-w_{1} U_{\xi \xi}-2(U-k \xi) U_{\xi}-U\left(U_{\xi}-k\right)=0, \quad \xi<X(t), \\
U(X(t), t)=0, \\
k X(t) \dot{X}(t)=w_{1,0} U_{\xi}, \quad \xi=X(t), \\
w_{1, \xi}=-k \xi+U, \quad \xi<X(t), \\
w_{1}(X(t), t)=w_{1,0}(0), \\
U(\xi, 0)=k \xi .
\end{array}
$$

This problem can be solved with a slight variation of the arguments in section 4 and the details will not be given here. Actually, if $k>0$ the argument given in Appendix $\mathrm{B}$ shows that the solution is global.

We now pass to describe the asymptotics of problem (4.2)-(4.6) as $\epsilon \rightarrow 0$. In the outer region, where $w_{1, x}$ does not develop large gradients, (4.3) can be approximated by the hyperbolic equation

$$
w_{1, t}+\left(c w_{1}\right)_{x}=0, x<\lambda(t) .
$$

This approximation is valid for $x$ at distances $O(1)$ of the free boundary $\lambda(t)$. Equation (5.27) has been analyzed in section 3, where we have seen that the solution develops a discontinuity at $x=0$ that grows exponentially. Since the solutions of the original free-boundary problem (4.2)-(4.6) do not develop discontinuities, a boundary layer is required near the free boundary $x=\lambda(t)$. For not very long times, we can understand the structure of such a boundary layer introducing the variables (5.12)-(5.14). In the diffusive boundary layer the behavior is controlled by the equations

$$
\begin{array}{r}
w_{1, t}-\left(\left(k \xi+w_{1, \xi}\right) w_{1}\right)_{\xi}=0, \quad \xi<X(t), \\
w_{1}(X(t), t)=w_{1,0}(0), \\
k X(t)+w_{1, \xi}=0, \quad \xi=X(t), \\
w_{1}(\xi, 0)=w_{1,0}(0) .
\end{array}
$$

The long time asymptotics of (5.28)-(5.31) can be analyzed by means of the change of variables

$$
w_{1}=e^{k t} G, \quad \xi=e^{\frac{k t}{2}} \eta, \quad X=e^{\frac{k t}{2}} Y
$$

which leads to

$$
\begin{array}{r}
\frac{\partial G}{\partial t}-\frac{3}{2} k \eta \frac{\partial G}{\partial \eta}-\frac{\partial}{\partial \eta}\left(G \frac{\partial G}{\partial \eta}\right)=0, \quad \eta<Y(t), \\
G(Y(t), t)=w_{1,0}(0) e^{-k t}, \\
k Y(t)+\frac{\partial G}{\partial \eta}(Y(t), t)=0 .
\end{array}
$$

The jump discontinuity for $w_{1}(x, t)$ in the outer region imposes on $G$ the following matching condition:

$$
G \sim w_{1,0}(0) \text { as } \eta \rightarrow-\infty .
$$

For times $t=O(1)$ the asymptotics of $w_{1}(x, t)$ near the free boundary is described by the solution $G$ of (5.33)-(5.36). Existence of global solutions can be discussed as in section 4.4. 
On the other hand, the long-time asymptotics of (5.33)-(5.36) can be described as follows. There exist several steady states of (5.33)-(5.35) satisfying the matching condition (5.36). The one which is relevant for our purposes is characterized by the fact that it is supported in $\left(-\infty, \eta_{0}\right)$ and satisfies

$$
G \sim \frac{3}{4} k \eta_{0}\left(\eta_{0}-\eta\right)
$$

The detailed description of the steady states of (5.33) is given in Appendix C.

Some steady states are positive in the whole line and some others are supported in a half line but behave like $C\left(\eta_{0}-\eta\right)^{\frac{1}{2}}$. In order to see why the only admissible steady state of (5.33)-(5.36) is the (unique) one with linear behavior near the extinction point $\eta_{0}$ we describe the boundary layer that appears near the point $\eta=\eta_{0}$ for (5.33)-(5.36).

Making the standard change of variables

$$
G=e^{-k t} W, \eta=\eta_{0}+e^{-k t} \nu
$$

problem (5.33)-(5.36) becomes, to leading order,

$$
\begin{aligned}
& \frac{3}{2} k \eta_{0} \frac{\partial W}{\partial \nu}+\frac{\partial}{\partial \nu}\left(W \frac{\partial W}{\partial \nu}\right)=0, \quad \nu<0, \\
& W=w_{1,0}(0) \text { at } \nu=0, \\
& \frac{\partial W}{\partial \nu}+k \eta_{0}=0 \text { at } \nu=0 .
\end{aligned}
$$

Integrating (5.39) and using (5.40), (5.41) we reduce the problem to the study of the first order equation

$$
\frac{3}{2} k \eta W+W \frac{\partial W}{\partial \nu}=\frac{k \eta_{0}}{2} w_{1,0}(0) .
$$

It is readily seen that this first order equation admits a monotonically decreasing solution which takes the value $w_{1,0}$ at $\nu=0$ and behaves at infinity as

$$
W \sim-\frac{3}{2} k \eta_{0} \nu, \text { as } \nu \rightarrow-\infty .
$$

Using (5.38) and (5.43), it follows that the inner behavior of the function $G$ described by the function $W(5.43)$ matches with the outer region if and only if the function $G$ has a linear behavior near $\eta_{0}$.

We should remark that a more refined argument is needed to exclude the case $\eta_{0}=0$. In this case (5.39) reduces to $W=w_{1,0}$. It turns out that there is a unique steady state of (5.33) satisfying the matching condition (5.36) supported in $(-\infty, 0)$ and behaving linearly near 0 . Matching between the outer and inner regions is not possible in this case, so that $\eta_{0}>0$.

The previous description of the boundary layer holds for times $t<<-\frac{1}{k} \ln (\sqrt{\epsilon})$. In order to describe the solution for larger times, we notice that the characteristics at distances $|x| \sim 1$ arrive to the region $|\eta| \sim 1$ for $t \sim \frac{1}{k}|\log (\sqrt{\epsilon})|$. For this range of times, the data of (5.33) for $|\eta|$ large, $\eta<0$, and $\eta \sim 1$ could be strongly oscillatory and depends basically on the initial data. Thus, the dynamics for these times is strongly dependent on the initial distribution $w_{1,0}(x, 0)$. For larger times, the asymptotics of the solutions depends on the asymptotics of $w_{1,0}$ as $x \rightarrow-\infty$. For instance, if 


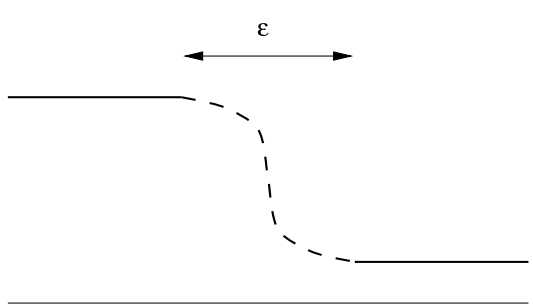

Classical regularization of a schock

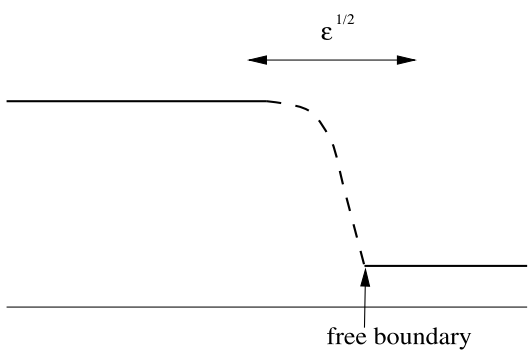

Free boundary regularization of (4.1), (4.2)

FIG. 5.1. Smoothing shocks out.

$w_{1,0}(x) \rightarrow w_{1, \infty}$ as $x \rightarrow-\infty$, we obtain that $w_{1}(x, t)$ grows exponentially. This is, however, rather unphysical, since it is due to a large flux of dislocations coming from $x=-\infty$.

We conclude with some final remarks. As Figure 5.1 shows, $w_{1}(x, t)$ corrects the solution of the conservation law we had for $\epsilon=0$, which is accurate out of an inner boundary layer of thickness $\sqrt{\epsilon}$ around $x=0$. In this inner boundary layer, the jump at $x=0$ observed in the solution for $\epsilon=0$ is smoothed out when $\epsilon>0$.

We may compare this problem with the regularization of shocks in usual scalar conservation laws by small diffusive terms (cf. [18]). The main difference is that, in our case, the diffusion acts only on one side of the shock and not throughout the shock (see Figure 5.1) Also, notice that the correction $u_{\epsilon}$ matches the left value of the jump with the value on the free boundary whereas for a shock in Burgers equation, for instance, it should join the left and right values of the jump. On the other hand, the length of the boundary layer is of order $\epsilon$ for Burgers equation while in our case it turns out to be of order $\sqrt{\epsilon}$.

6. Conclusions. We have introduced some simple one dimensional models of dislocation dynamics that, despite their simplicity, exhibit some features that are experimentally observed in the evolution of families of dislocations. The phenomena we have been interested in describing are those of pattern formation (associated with the loss of hyperbolicity in our models), and the related pile-up of dislocations due to inhomogeneous pinning distributions.

We have considered two types of models. In the first of them, involving mutual interactions between two families of dislocations, the equations are conservation laws of mixed type. The second model, in which one family of dislocations is externally pinned, is a degenerate nonlinear parabolic system of equations that, in particular cases, reduces to a free-boundary problem.

We have discussed the well-posedness of those models. The solutions of the mixed system of conservation laws develop singularities for some initial data that are smoothed out in the regularized parabolic problem. We have described in a detailed way how this regularization takes place.

We observe that we have obtained two different forms of pattern formation. In the mode that we can call hyperbolic, we have obtained piling up of dislocations at the particular points where pinning forces are strong enough to stop the motion of dislocations. On the other hand, when both families of dislocations are free to move we find small perturbations of uniform states are unstable, which should lead to banding. To see whether our simple models can reproduce the honeycomb structures 
seen experimentally we will need to regularize the coupled (2.9)-(2.13) model in the same way that we have regularized the single-moving-dislocation-family model (2.14). However, even if this is the case and the model evolves to a situation in which there is no relative motion between the dislocation families due to tangling, the honeycomb structure will not be fixed in space without the inclusion of an additional external pinning force.

Appendix A. Self-similar solution. As stated in section 4, problem (4.19)(4.21) admits a self-similar solution with increasing $\lambda(t)$ in the form (4.22)-(4.24). It follows from (4.19), (4.20), (4.21) that the function $\phi$ in (4.22) satisfies

$$
\begin{array}{rr}
\phi_{y y}+\frac{y \phi}{2}-\frac{1}{2} \phi=0, & y<\xi_{0}, \\
\phi\left(\xi_{0}\right)=0, & \\
\phi_{y}\left(\xi_{0}\right)=\frac{\xi_{0}^{2} \operatorname{sign}(\Gamma)}{2}, \\
\phi(y) \rightarrow \Gamma y, \quad y \rightarrow-\infty .
\end{array}
$$

The solution of (A.1), (A.2) is given by

$$
\phi(y)=\Gamma y+K y \int_{-\infty}^{y} \frac{e^{-\frac{s^{2}}{4}}}{s^{2}} d s .
$$

It is not hard to check that the function $\phi(y)$ is analytic in the whole complex plane, since the singularity of the integral is cancelled out by the factor $y$. From now on, we will understand that the integral in (A.5) is a meromorphic function in the complex plane with a pole at $y=0$.

The values of $K$ and $\xi_{0}$ must be chosen in order to satisfy the boundary conditions (A.2), (A.3):

$$
\Gamma+K \int_{-\infty}^{\xi_{0}} \frac{e^{-\frac{s^{2}}{4}}}{s^{2}} d s=0, K \frac{e^{-\frac{\xi_{0}^{2}}{4}}}{\xi_{0}}=\frac{\xi_{0}^{2} \operatorname{sign}(\Gamma)}{2} .
$$

Since we are interested in solutions with $\lambda^{\prime}(t)>0$, it follows that $\xi_{0}>0$. Eliminating $K$ from (A.6) we get for $\xi_{0}$ the equation

$$
|\Gamma|+\frac{\left(\xi_{0}\right)^{3} e^{\frac{\xi_{0}^{2}}{4}}}{2} \int_{-\infty}^{\xi_{0}} \frac{e^{\frac{-s^{2}}{4}}}{s^{2}} d s=0 .
$$

To get rid of the singularity of the integral terms, it is convenient to rewrite (A.7) as follows:

$$
|\Gamma|=\frac{\left(\xi_{0}\right)^{3} e^{\frac{\xi_{0}^{2}}{4}}}{2}\left(\frac{1}{\xi_{0}}+\int_{-\infty}^{\xi_{0}} \frac{1-e^{-\frac{s^{2}}{4}}}{s^{2}} d s\right)=B\left(\xi_{0}\right) .
$$

The function $B\left(\xi_{0}\right)$ is monotonically increasing and satisfies

$$
\begin{aligned}
B\left(\xi_{0}\right) \sim \frac{\xi_{0}^{2}}{2}, & \xi_{0} \rightarrow 0^{+}, \\
B\left(\xi_{0}\right) \sim \alpha \frac{\xi_{0}^{3} e^{\frac{\xi_{0}^{2}}{4}}}{2}, & \xi_{0} \rightarrow \infty,
\end{aligned}
$$

where $\alpha=\int_{-\infty}^{\infty} \frac{1-e^{-\frac{s^{2}}{4}}}{s^{2}} d s>0$. Therefore, there exists a unique solution $\xi_{0}$ to (A.8) in the interval $(0, \infty)$. Then, the value of $K$ is computed using (A.6). 
This self-similar solution provides the local asymptotics of the solutions of the original free-boundary problem (4.2)-(4.6) as $t \rightarrow 0^{+}$.

Appendix B. Proof of Theorem 4.1. As we stated in section 4.4 the main idea in the proof is to approximate problem (4.2)-(4.6) by the sequence of regularized problems (4.27)-(4.30) with $\Omega_{0}(x, t)$ given by

$$
\begin{array}{r}
\Omega_{0}(x, t)=w_{1,0}(\tilde{u}(t))+\frac{1}{\epsilon} \int_{\tilde{u}(t)}^{x} d \eta\left(c(\eta)+\Gamma \sqrt{\epsilon w_{1,0}(\lambda(0)) t}\right. \\
\left.\cdot \phi\left(\frac{\eta-\lambda(0)}{\sqrt{\epsilon w_{1,0}(\lambda(0)) t}}\right)+\Gamma \sqrt{\epsilon w_{1,0}(\lambda(0)) t} \nu\left(\frac{\eta-\lambda(0)}{\sqrt{\epsilon w_{1,0}(\lambda(0)) t}}\right)\right) .
\end{array}
$$

Here, $\phi$ is the profile of the self-similar solution (cf. (4.22), (4.23)) and $\nu(x)=u(x, 0)-$ $\Gamma(x-\lambda(0))$, for $x<\lambda(0)$. For $x>\lambda(0), \nu=0$. Using (B.1), we deduce

$$
\left|\Omega_{0}\left(x, t_{n}\right)-w_{1,0}(x)\right| \leq C t_{n} .
$$

We aim to solve the approximated problems (4.27), (4.28), (4.29), (4.30) for a time interval independent of $n$ and obtain uniform bounds on the solutions in such a time interval. More precisely, we have the following local existence result.

Proposition B.1. If $c \in C^{2}$ is globally bounded and $w_{1,0} \in C^{2, \alpha}$ with $0<\frac{1}{K}<$ $w_{1,0}<K$ there exists a unique solution $w_{1, n}$ of problem (4.27), (4.28), (4.29), (4.30) in a time interval $\left[t_{n}, t_{n}+\delta(\epsilon)\right]$, where $\delta(\epsilon)$ is independent on $n$.

We will prove Proposition B.1 in two steps. First, we will obtain a solution of (4.27)-(4.30) in a time interval $\left[t_{n}, t_{n}+\delta_{n}\right]$ depending on $n$. This is done by means of a slight modification of a classical Stefan problem (cf. [9]). As a next step, we will prove by means of suitable sub- and supersolutions that the time of existence is independent of $n$.

Lemma B.2. Under the asumptions of Proposition B.1, there exists a unique solution $w_{1, n}, \lambda_{n}$ to (4.27)-(4.30) for a time interval $\left[t_{n}, t_{n}+\delta_{n}\right]$. The free boundary is $C^{1, \alpha}\left(\left[t_{n}, t_{n}+\delta_{n}\right]\right)$.

Proof. We introduce the following space of functions $X_{n}=\left\{\lambda \in C^{1, \alpha}\left[t_{n}, t_{n}+\right.\right.$ $\left.\left.\delta_{n}\right], \lambda\left(t_{n}\right)=\hat{\lambda}\left(t_{n}\right),\|\lambda\|_{\alpha}<M, \frac{1}{2} \hat{\lambda}(t)<\lambda(t)<2 \hat{\lambda}(t)\right\}$. We intend to make a fixed point in this space of functions. To this end, we solve the boundary value problem (4.27), (4.29), (4.30) for any $\lambda(.) \in X_{n}$. In order to obtain some a priori estimates for $w_{1, n}$ we use suitable sub and supersolutions. Let us define $\tilde{w}_{1}(x)$ as the solution of

$$
\epsilon \frac{\partial \tilde{w}_{1}}{\partial x}=c(x), \quad \tilde{w}_{1}(\bar{\lambda}(0))=M,
$$

where $M>0$ will be chosen later.

It is straightforward to check that $\frac{M}{2} \leq \tilde{w}_{1} \leq \frac{3}{2} M$ in a neighborhood $(\bar{\lambda}(0)-$ $\left.\eta_{0}(\epsilon), \bar{\lambda}(0)+\eta_{0}(\epsilon)\right)$, where $\eta_{0}(\epsilon)>0$ is independent of $n$. We truncate $\tilde{w}_{1}$ in a smooth way in order to make it constant and positive for $x<\bar{\lambda}(0)-\eta_{0}(\epsilon)$. Let us denote by $\bar{w}_{1}$ the resulting truncated function. Moreover, we can make this truncation in such a way that

$$
\left|\frac{\partial}{\partial x}\left(\left(c(x)-\epsilon \frac{\partial \bar{w}_{1}}{\partial x}\right) \bar{w}_{1}\right)\right| \leq C(M, \epsilon) \bar{w}_{1}
$$

is satisfied. 
We define $\hat{w}_{1}(x, t)=\bar{w}_{1}(x) e^{L t}$. Picking up $L$ and $M$ in a suitable way we obtain that $\hat{w}_{1}$ is a sub- or a supersolution for (4.27), (4.29), (4.30).

Set $u_{n}(x, t)=\epsilon \frac{\partial w_{1, n}}{\partial x}-c(x)$. It is readily checked that $u_{n}$ solves

$$
\begin{aligned}
u_{n, t}-\epsilon w_{1, n} \frac{\partial^{2} u_{n}}{\partial x^{2}}-2\left(u_{n}+c\right) \frac{\partial u_{n}}{\partial x}-u_{n}\left(\frac{\partial u_{n}}{\partial x}+c^{\prime}\right) & =0, x<\lambda_{n}(t) \\
u & =0, x=\lambda_{n}(t)
\end{aligned}
$$

Formally differentiating (4.28), we obtain

$$
u(x, 0) \lambda^{\prime}(t)=\epsilon w_{1,0}(x) \frac{\partial u}{\partial x}(x, t), x=\lambda(t) .
$$

Obviously, given an arbitrary function $\lambda_{n}(t) \in X_{n}$, condition (B.7) is not satisfied. However, we intend to choose $\lambda_{n}(t)$ verifying (B.7) by means of a fixed point argument.

Taking into account $u(x, 0) \approx \Gamma(x-\lambda(0))+O\left((x-\lambda(0))^{2}\right)$ we can check that $u_{n}(\lambda(t), 0) \neq 0$ for any $\lambda \in X_{n}$. We then rewrite (B.7) in an integral form

$$
\lambda(t)=\bar{\lambda}\left(t_{n}\right)+\int_{t_{n}}^{t} \epsilon w_{1,0}(\lambda(t)) \frac{\partial u}{\partial x}(\lambda(s), s) d s=T(\lambda(t)) .
$$

Classical regularity theory for parabolic equations shows that $\frac{\partial u}{\partial x}(\lambda(t), t) \in C_{t}^{\frac{1}{2}+\frac{\alpha}{2}}\left[t_{n}\right.$, $\left.t_{n}+\delta_{n}\right]$. Actually, we have uniform bounds for this derivative depending only on the initial data as well as $M$. From this, we inmediatedly deduce that the operator $T(\lambda(t))$ has a fixed point for $\delta_{n}$ small enough by means of a standard contractive fixed point argument. Since the argument is well known for the classical Stefan problem we shall not provide more details here.

Notice that we have obtained a solution $w_{1, n}$ of (4.27), (4.29), (4.30) satisfying (B.7). It only remains to check that condition (4.28) holds. To this end, notice that (B.7) implies $\frac{\partial}{\partial t}\left(w_{1, n}\left(\lambda(t)^{-}, t\right)-w_{1,0}(\lambda(t))\right)=0$, whence $w_{1, n}\left(\lambda(t)^{-}, t\right)=w_{1,0}(\lambda(t))+$ $K$. Evaluating this expression for $t=t_{n}$ and taking into account (B.1), we deduce that $K=0$. Thus, (4.28) follows.

Remark. As a subproduct of the proof of Lemma B.2 we have obtained

$$
0<\frac{1}{M^{\prime}} \leq w_{1, n} \leq M^{\prime}, \quad t \in\left[t_{n}, t_{n}+\delta_{n}\right] .
$$

Actually, the argument shows that this estimate is satisfied as far as $w_{1, n}$ is defined if $t \leq \delta(\epsilon)$, where $\delta(\epsilon)$ is a suitable number independent of $\epsilon$.

Lemma B.3. Under the assumptions of Proposition B.1, the following estimates hold in $\left[t_{n}, t_{n}+\delta_{n}\right]$ for $x<\min \left(\lambda_{n}(t), \hat{\lambda}(t)\right)$ :

$$
\begin{aligned}
\left|u_{n}(x, t)-\hat{u}(x, t)\right| \leq & C\left((x-\lambda(0))^{2}+t\right), \\
\left|\frac{\partial u_{n}}{\partial x}(x, t)-\frac{\partial \hat{u}}{\partial x}(x, t)\right| \leq & C(|x-\lambda(0)|+\sqrt{t}), \\
& \left|\lambda_{n}(t)-\hat{\lambda}(t)\right| \leq C t,
\end{aligned}
$$

where $\hat{u}$ and $\hat{\lambda}$ correspond to the self-similar solution (4.22), (4.23) and $u_{n}, \lambda_{n}$ are the solutions constructed in Lemma B.2. The constant $C$ does not depend on $n$, although it could depend on $\epsilon$. 
Proof. By convenience, we introduce a new set of variables as follows:

$$
\begin{array}{r}
u=\Gamma \sqrt{\epsilon w_{1,0}(\lambda(0))} \bar{u}, x=\lambda(0)+\sqrt{\epsilon w_{1,0}(\lambda(0))} \bar{x}, \\
\lambda(t)=\lambda(0)+\sqrt{\epsilon w_{1,0}(\lambda(0))} \bar{x}(t) .
\end{array}
$$

In this way, problem (5.1)-(5.4) becomes

$$
\begin{array}{r}
\bar{u}-\frac{w_{1}}{w_{1,0}(\lambda(0))} \bar{u}_{\overline{x x}}-2\left(\Gamma \bar{u}+\frac{c(x)}{\sqrt{\epsilon w_{1,0}(\lambda(0))}}\right) \bar{u}_{\bar{x}}-\bar{u}\left(\Gamma \bar{u}_{\bar{x}}+c\right)=0, \quad \bar{x}<\bar{\lambda}(t), \\
\bar{u}=0 \text { at } \bar{x}=\bar{\lambda}(t), \\
\bar{u}(\bar{x}, 0) \bar{\lambda}^{\prime}(t)=\frac{w_{1,0}}{w_{1,0}(\lambda(0))} \frac{\partial \bar{u}}{\partial \bar{x}} \text { at } \bar{x}=\bar{\lambda}(t), \\
\bar{u}(\bar{x}, 0)=\bar{x}+O\left(\sqrt{\epsilon} \bar{x}^{2}\right), \quad \bar{x} \longrightarrow 0 .
\end{array}
$$

The self-similar solution given in (4.22), (4.23) can be written as

$$
\tilde{u}=\sqrt{t} \phi\left(\frac{\bar{x}}{\sqrt{t}}\right), \bar{\lambda}(t)=\xi_{0} \sqrt{t} .
$$

We define the function $v_{n}(x, t)=\bar{u}_{n}(\bar{x}, t)-\tilde{u}(\bar{x}, t)$. From now on, we will drop the index $n$ for ease of notation. Simple (but tedious) computations show that $v$ solves

$$
\begin{array}{r}
v_{t}=(1+h(x, t)) v_{\overline{x x}}+h(x, t) \tilde{u}_{\overline{x x}}+F, \quad \bar{x}<\bar{\lambda}(t), \\
v(\bar{\lambda}(t), t)=\tilde{u}(\tilde{\lambda}(t), t)-\bar{u}(\bar{\lambda}(t), t), \\
\left(\bar{\lambda}(t)+\Gamma \sqrt{\epsilon w_{1,0}(\lambda(0))} \nu(\bar{\lambda}(t))\right) \bar{\lambda}^{\prime}(t) \\
=\frac{w_{1,0}(x)}{\Gamma w_{1,0}(\lambda(0))}\left(\tilde{u}_{\bar{x}}(\bar{\lambda}(t), t)+v_{\bar{x}}(\bar{\lambda}(t), t)\right), \\
v\left(x, t_{n}\right)=\sqrt{\epsilon w_{1,0}(\lambda(0))} \nu(\bar{x})
\end{array}
$$

with

$$
\begin{aligned}
F= & 2\left(\Gamma \sqrt{\epsilon w_{1,0}(\lambda(0))} \tilde{u}(\bar{x}, t)+\Gamma \sqrt{\epsilon w_{1,0}(\lambda(0))} v(\bar{x}, t)+c(x)\right) \tilde{u}_{\bar{x}} \\
& +2\left(\Gamma \sqrt{\epsilon w_{1,0}(\lambda(0))} \tilde{u}(\bar{x}, t)+\Gamma \sqrt{\epsilon w_{1,0}(\lambda(0))} v(\bar{x}, t)+c(x)\right) v_{\bar{x}} \\
& +\sqrt{\epsilon w_{1,0}(\lambda(0))}(\tilde{u}(\bar{x}, t)+v(\bar{x}, t))\left(\Gamma \tilde{u}_{\bar{x}}(\bar{x}, t)+\Gamma v_{\bar{x}}(\bar{x}, t)+c^{\prime}(x)\right)
\end{aligned}
$$

and $h(x, t)=\frac{w_{1}(x, t)}{w_{1,0}(\lambda(0))}-1$.

We now use a standard bootstrap argument. We assume

$$
\begin{array}{r}
\left|w_{1}(x, t)-w_{1,0}(\lambda(0))\right| \leq M(\sqrt{t}+|x-\lambda(0)|), \\
|\bar{\lambda}(t)| \leq M \sqrt{t}
\end{array}
$$

with $M$ large, independent of $n$ but not of $\epsilon$. Using (B.22), (B.23) we are able to control the nonlinear terms in (B.18)-(B.21). Notice that (B.22), (B.23) hold for 
the initial data. The analysis of (B.18)-(B.21) taking into account these bounds will provide (B.22), (B.23) with the constant $M$ replaced by a constant $C$ independent of $M, n$ for short times.

After some computations, we get that, as far as (B.22), (B.23) hold, the following inequalities are satisfied:

$$
\begin{aligned}
|h(x, t)| \leq C M(\sqrt{t}+|x-\lambda(0)|), & \sqrt{t}+|x-\lambda(0)| \leq R_{0}, \\
|F(x, t)| \leq C, & \sqrt{t}+|x-\lambda(0)| \leq R_{0} .
\end{aligned}
$$

From now on, $C$ will be a positive constant that can change from line to line but is independent on $n, M$, although it can depend on $\epsilon$. The constant $R_{0}$ in (B.24), (B.25) is also independent of $n, M$.

Now, we intend to obtain sub- and supersolutions for the problem (B.18)-(B.21) in a self-similar form. To this end, we write

$$
\begin{array}{r}
v=t Q(y, \tau), \quad y=\frac{\bar{x}}{\sqrt{t}}, \tau=\log (t), \\
\bar{\lambda}(t)=\tilde{\lambda}(t)+t \nu(\tau),
\end{array}
$$

where $\tilde{\lambda}$ is as in (B.17). In this new set of variables, (B.18) becomes

$$
Q_{\tau}=Q_{y y}-\frac{y}{2} Q_{y}+Q+h(x, t) Q_{y y}+\frac{h(x, t)}{\sqrt{(} t)} \phi_{y y}+F .
$$

The Dirichlet boundary condition becomes

$$
Q\left(\xi_{0}+\nu(\tau) \sqrt{t}, \tau\right)=\frac{-\phi\left(\xi_{0}+\nu(\tau) \sqrt{t}\right)+\phi \xi_{0}}{\sqrt{t}} .
$$

We will check later that we can use comparison arguments for the free-boundary problem (B.18)-(B.21) if the equation (B.20) is satisfied with the identity sign replaced by inequalities. More precisely, for supersolutions we have to put the sign $\leq$ in (B.20) and for subsolutions the sign $\geq$. In the variable $Q$, these inequalities for sub- and supersolutions become

$$
\begin{aligned}
\xi_{0}\left(\nu_{0}+\nu\right)+\frac{\xi_{0}}{2} \nu \geq(\leq) & \left(1+f_{1}(\tau)\right) \frac{\partial Q}{\partial y}+\frac{\xi_{0}}{w_{1,0}(\lambda(0))} \frac{\partial w_{1,0}}{\partial x}(\lambda(0)) \phi_{y}(\xi) \\
& -\frac{\xi_{0}}{2} \Gamma \sqrt{\xi w_{1,0}(\lambda(0))} \frac{v(\tilde{\lambda}(t))}{t}+f_{2}(t)
\end{aligned}
$$

at $y=\xi_{0}+\sqrt{t} \nu(\tau)$, where $\left|f_{1}(\tau)\right| \leq C \sqrt{t},\left|f_{2}(\tau)\right| \leq C M \sqrt{t}$ and $v$ as in (B.18). Notice that

$$
\left|\frac{\xi_{0}}{w_{1,0}(\lambda(0))} \frac{\partial w_{1,0}}{\partial x}(\lambda(0)) \phi_{y}(\xi)-\frac{\xi_{0}}{2} \Gamma \sqrt{\xi w_{1,0}(\lambda(0))} \frac{v(\tilde{\lambda}(t))}{t}\right| \leq \beta,
$$

where $\beta$ is a constant independent of $n$.

We look for sub- and supersolutions of (B.28) with the form

$$
Q(y, \tau)=G(y)+W(y, \tau)
$$


where $G$ and $W$ will be determined later. Roughly speaking the free boundary for these super- and subsolutions will be placed at $y \approx \xi_{0}+\nu_{0} \sqrt{t}$, where $\nu_{0}$ is a suitable constant to be determined.

Let us define $\tilde{G}(y)$ as the unique solution of

$$
\begin{array}{r}
\tilde{G}_{y y}+\frac{y}{2} \tilde{G}_{y}-\tilde{G}=0, \\
\tilde{G}\left(\xi_{0}\right)=\frac{\xi_{0}}{2}, \quad \tilde{G}_{y}\left(\xi_{0}\right)=-\left(\frac{3}{2} \xi_{0}+\frac{\xi_{0}^{3}}{4}\right),
\end{array}
$$

and $\bar{G}$ the unique solution of problem

$$
\begin{array}{r}
\bar{G}_{y y}+\frac{y}{2} \bar{G}_{y}-\bar{G}=C M(1+|y|)\left|\phi_{y y}\right|+C, \\
\bar{G}\left(\xi_{0}\right)=\bar{G}_{y}\left(\xi_{0}\right)=0,
\end{array}
$$

where the constant $C$ is chosen as in (B.24), (B.25). It is readily seen that $\tilde{G}$ is a monotonically decreasing function that grows quadratically as $y \rightarrow-\infty$. On the other hand, $|\bar{G}| \leq C M\left(1+y^{2}\right)$.

Finally, we define a function $\hat{G}$ as the solution of the following problem:

$$
\begin{array}{r}
\hat{G}_{y y}+\frac{y}{2} \hat{G}_{y}-\hat{G}=0, \quad y<\xi_{0}, \\
\hat{G}\left(\xi_{0}\right)=0, \hat{G}_{y}\left(\xi_{0}\right)= \pm \beta,
\end{array}
$$

where $\beta$ is as in (B.30) and the signs \pm are chosen for super- and subsolutions, respectively. We choose $G$ in (B.31) as

$$
G=-\nu_{0} \tilde{G}+\bar{G}+\hat{G}
$$

Taking $\nu_{0}$ large enough (depending on $M$ ) we can consider that $\bar{G}, \hat{G}$ in (B.38) are small perturbations compared to $-\nu_{0} \tilde{G}$. In particular (and depending on the sign of $\nu_{0}$ ), we can assume that $G$ is either strictly positive or negative.

We define the function $\nu(\tau)$ by means of the Dirichlet boundary condition (B.29). Using the implicit function theorem it is not hard to check that there exists a function $\nu$ solving (B.29) and satisfying

$$
\left|\nu(\tau)-\nu_{0}\right| \leq C M \sqrt{t},\left|\nu_{\tau}\right| \leq C M \sqrt{t} .
$$

We now proceed to determine the function $W$ in (B.31). In order to ensure that $Q$ is a subsolution (resp., supersolution) of problem (B.28), (B.29), (B.20), $W$ must be a subsolution (resp., supersolution) for problem

$$
\begin{array}{r}
W_{\tau}-\frac{y}{2} y W_{y}+W-(1+h) W_{y y}=h\left|G_{y y}\right|, \\
W\left(\xi_{0}+\nu(\tau) \sqrt{t}, \tau\right)=0 .
\end{array}
$$

The boundary condition (B.30) becomes, after using (B.33), (B.35), (B.36), and (B.39),

$$
\xi_{0} \nu_{\tau} \geq(\leq)\left(\frac{\partial W}{\partial y}+f_{3}(\tau)\right)
$$

where $\left|f_{3}(\tau)\right| \leq C M \sqrt{t}$. 
We split $W=W_{1}+W_{2}$, where $W_{1}$ will control the right-hand side in (B.40) and $W_{2}$ will take care of the free-boundary condition (B.41). We need to choose $W_{1}$ verifying

$$
\begin{array}{r}
W_{1, \tau}-\frac{y}{2} y W_{1, y}+W_{1}-(1+h) W_{1, y y} \geq(\leq)|h|\left|G_{y y}\right|, \\
W_{1}\left(\xi_{0}+\nu(\tau) \sqrt{t}, \tau\right)=0 .
\end{array}
$$

Taking into account (B.38) it is easily seen that $\left|G_{y y}\right| \leq C M$. Using this fact and (B.24) we can obtain a function $W_{1}$ satisfying (B.40), (B.41) and

$$
\left|W_{1}\right| \leq C(M) \sqrt{t}(1+\sqrt{y})
$$

for $0 \leq t \leq R_{0}$, with $R_{0}$ independent on $n$. The construction of $W_{1}$ is achieved by using sub- and supersolutions of the form $\tilde{W}_{1}=e^{\frac{\tau}{2}} S(y)$ where $S$ is a positive solution of

$$
S_{y y}+\frac{y}{2} S_{y}-\frac{3}{2} S=C(M)(1+|y|)
$$

verifying $|S| \leq C(M)(1+|y|)$. From (B.45) and standard regularizing effects for parabolic equations we deduce that

$$
\left|W_{1, y}\right| \leq C(M) \sqrt{t}
$$

at the free boundary $y=\xi_{0}+\nu(\tau) \sqrt{t}$.

Finally, we define $W_{2}$ as a solution of the boundary value problem

$$
\begin{aligned}
W_{2, \tau}-\frac{y}{2} y W_{2, y}+W_{2}-h W_{2, y y} & =0, \\
W_{2}\left(\xi_{0}+\nu(\tau) \sqrt{t}, \tau\right) & =0 .
\end{aligned}
$$

Actually, we want to select $W_{2}$ in such a way that (B.42) holds. Taking into account (B.29), (B.47) and the bound on $f_{3}$, it turns out that in order to obtain (B.42) we just need to have $W_{2}$ such that

$$
W_{2, y}\left(\xi_{0}+\nu(\tau) \sqrt{t}, \tau\right) \sim \pm A \sqrt{t},
$$

where $A$ is a constant depending on $M$. This can be achieved solving

$$
\psi_{t}=(1+h) \psi_{x x}, \quad \psi(x, 0)= \pm \min \left(\tilde{A} x^{3}, 1\right)
$$

in $x \leq \xi \sqrt{t}+\nu(\tau) t$, with $\tilde{A}$ large enough. Defining $W_{2}$ in such a way that $\psi(x, t)=$ $t^{\underline{3}} W_{2}(y, \tau)$ we obtain (B.50).

Notice that the functions $Q$ we have constructed so far are sub- and supersolutions just in a neighborhood of the origin. In order to estimate the solutions away from the origin we can just take into account (5.43), (B.47) and standard regularizing effects for parabolic equations. We then obtain that $v$ is bounded in the line $x=-X_{0}$. Here, $X_{0}$ is a small number independent on $n$, chosen in such a way that $Q$ is a sub-supersolution for $x \geq-X_{0}$. We can then add to the function $Q$ an additional term $R(x, t)$ that solves (B.18), vanishes for $t=0$, controls the boundary values of $v$ in $x=-X_{0}$, and vanishes along the curves $x=\xi_{0} \sqrt{t}+\nu(\tau) t$. Standard estimates for the Green function of parabolic equations with bounded coefficients (cf. [2]) imply that $\left|R_{y}\right| \leq C e^{-\frac{\beta}{t}}$ for some $\beta>0$. 


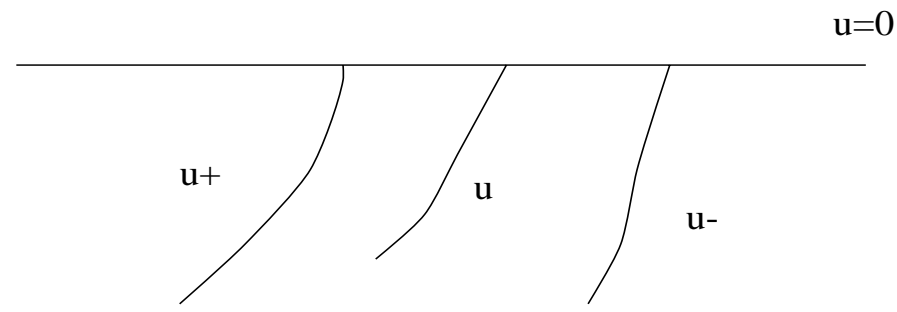

FIG. B.1. Maximum principle.

It is not hard to check that after a slight modification (if needed) of the function $W_{2}, Q+R$ is a sub-supersolution satisfying (B.28), (B.29), (B.30).

We now show how the maximun principle works for these type of free-boundary problems. It can be checked that for short times, the most important term in the suband supersolution $Q$ is the function $-\nu_{0} \tilde{G}(y)$.

Notice that, in the original set of variables (B.12) the aspect of super- and subsolutions compared to $u$ is the one given in Figure B.1. It is important to take into account that both the sub- and the supersolution vanish at their free boundaries due to (B.29).

On the other hand, as far as $\lambda^{+} \leq \bar{\lambda} \leq \lambda^{-}$we can use the classical maximum principle to show that

$$
u^{-} \leq \bar{u} \leq u^{+}
$$

in any common region of validity. If at some particular time, there is an intersection between $\bar{\lambda}$ and $\lambda^{+}$(resp., $\lambda^{-}$), it follows from (B.52) that $\frac{\partial \bar{u}}{\partial x} \geq \frac{\partial u^{+}}{\partial x}$ (resp., $\frac{\partial \bar{u}}{\partial x} \leq \frac{\partial u^{-}}{\partial x}$ ). Taking into account (B.30) it then follows that $\frac{d \lambda^{+}}{d t}<\frac{d \bar{\lambda}}{d t}$ (resp., $\frac{d \lambda^{-}}{d t}>\frac{d \bar{\lambda}}{d t}$ ). It then follows that $\bar{\lambda}$ remains between $\lambda^{+}$and $\lambda^{-}$as far as the solution is defined. Therefore, (B.11) follows.

On the other hand, we have obtained

$$
\begin{array}{r}
v_{n}(\bar{x}, t) \geq C_{M} t\left(1+\left(\frac{\bar{x}}{\sqrt{t}}\right)^{2}\right), \quad \bar{x} \geq \bar{\lambda}(t)-2 \nu_{0} t \\
v_{n}(\bar{x}, t) \geq-C_{M} t\left(1+\left(\frac{\bar{x}}{\sqrt{t}}\right)^{2}\right), \quad \bar{x} \leq \bar{\lambda}(t) .
\end{array}
$$

We can extend the region of validity of (B.53), (B.54) taking into account that, by (B.11), $v$ is linearly bounded by $t$ at the free boundary and using a linear supersolution (in $t$ ). This proves (B.9).

Finally, (B.10) follows by means of a rescaling, as well as classical regularizing effects. Integrating (B.9), we obtain

$$
\left|w_{1}(x, t)-w_{1,0}(\lambda(0))\right| \leq C(\sqrt{t}+|x|)|\lambda(t)| \leq 2|\bar{\lambda}(t)|,
$$

where $C$ can be chosen independently on $M$ if $t$ is small enough (and also on $n$ ). This concludes the bootstrap argument that we began in (B.24), (B.25).

End of the proof of Proposition B.1. In order to prove uniformity in $n$ for the time of existence of the solutions we use (as usual for the classical Stefan problem) suitable 
sub- and supersolutions. To this end, we first transform the free-boundary problem in a fixed-boundary problem by means of the change of variables $y=\tilde{y}+\sqrt{t} \nu(\tau)$.

It is not hard to construct sub- and supersolutions for (B.13)-(B.16) in the form $\bar{u}=\sqrt{t} H(\bar{y})$, where $H$ satisfies

$$
-\frac{\partial^{2}}{\partial y^{2}} H-\frac{\bar{y}}{2} \frac{\partial H}{\partial \bar{y}}+\frac{H}{2} \geq(\leq) \epsilon_{0} \frac{\partial H}{\partial \bar{y}}
$$

where $\epsilon_{0}$ is a small positive number and $t$ is small enough (independent on $n$ ). We then obtain in this form that

$$
|\dot{\lambda}(t)| \leq \frac{C}{\sqrt{t}}
$$

Combining (B.9), (B.11), and (B.57) we obtain by means of a rescaling and classical parabolic estimates (B.10).

End of the proof of Theorem 4.1. A standard compactness argument shows that the sequence $\left(u_{n}, \lambda_{n}\right)$ converges as $n \rightarrow \infty$ to a function $(u, \lambda)$ which solves (4.14)(4.16). Notice that the initial data, as well as the free-boundary condition, pass to the limit due to (B.9), (B.10), (B.11), which hold for a time $0 \leq t \leq \delta(\epsilon)$.

It only remains to show that the solution so far obtained is globally defined in time. Taking into account the assumption (4.26) it follows (using exponential suband supersolutions) that the function $w_{1}(x, t)$ satisfies $0 \leq \frac{1}{C(T)} \leq w_{1}(x, t) \leq C(T)$ for $0 \leq t \leq T$. As for the classical Stefan problem, it is enough to check that, if $|\dot{\lambda}(t)| \leq C(T)$ for $0 \leq t \leq T$. This is done by comparison with a subsolution. Combining (4.25) and (4.15), (4.16) we have that $\dot{\lambda}(t)>0$.

It is convenient to transform the problem in a fixed free-boundary problem by means of the change of variables $x=\lambda(t)+\epsilon$.

In this new set of variables we can construct a subsolution for (4.14)-(4.16) in the form

$$
\bar{u}=C_{1} \xi+C_{2} \xi^{2}
$$

for $\xi<0$ close to 0 and then extending by a constant. Since $u$ is bounded in compact sets, away from the free boundary, by classical regularizing effects we can use (B.58) as a subsolution for our problem. By comparison $\frac{\partial u}{\partial x} \leq C(T)$ for $0 \leq t \leq C(T)$. We do not insist on the details since the argument is standard.

Appendix C. Phase portrait. In this section, we study the solutions of the ordinary differential equation

$$
\eta G_{\eta}+G G_{\eta \eta}+G_{\eta}^{2}=0
$$

which are the steady states of (5.33). We recall that in section 5 we were interested in solutions of (C.1) satisfying the matching condition (5.36). Therefore, we will consider solutions of (C.1) satisfying

$$
G(\eta) \rightarrow k, \quad \eta \rightarrow-\infty
$$

where $k$ is a positive constant. Moreover, by the analysis in section 5 , the function $G$ should be supported in a half line. 
Standard local analysis for ordinary differential equations shows that for each constant $C \in R$ there exists a unique solution of (C.1) satisfying

$$
G \sim k+C \frac{e^{-\frac{\eta^{2}}{2 k}}}{|\eta|}, \quad \eta \rightarrow-\infty .
$$

It readily follows from (C.1) that $G$ is a strictly monotonic function unless it is a constant. On the other hand, since $G$ must vanish at a finite $\eta_{0} \in R$ we should take $C<0$ in (C.3).

In order to describe the solutions of (C.1) it is convenient to transform the equation into an autonomous system by means of the change of variables

$$
\eta=\operatorname{sign}(\eta) e^{\tau}, \quad G=\eta^{2} W, \quad Z=2 W+\eta W_{\eta} .
$$

In these variables, (C.1) becomes

$$
\begin{array}{r}
Z_{\tau}=-Z-\frac{Z+Z^{2}}{W}=-\frac{\left(Z W+Z+Z^{2}\right)}{W}, \\
W_{\tau}=Z-2 W .
\end{array}
$$

We remark that system (C.5), (C.6) is independent of the sign of $\eta$ although the change of variables (C.4) depends on it.

Since $G_{\eta}=\eta Z$ and by assumption we restrict to solutions with $G_{\eta}<0$ we need to analyze system (C.5), (C.6) in the region $Z>0$ if $\eta>0$ and $Z<0$ if $\eta<0$. On the other hand, $G>0$ implies $W>0$.

Standard analysis shows that the phase portrait associated to system (C.5), (C.6) is the one in Figure C.1.

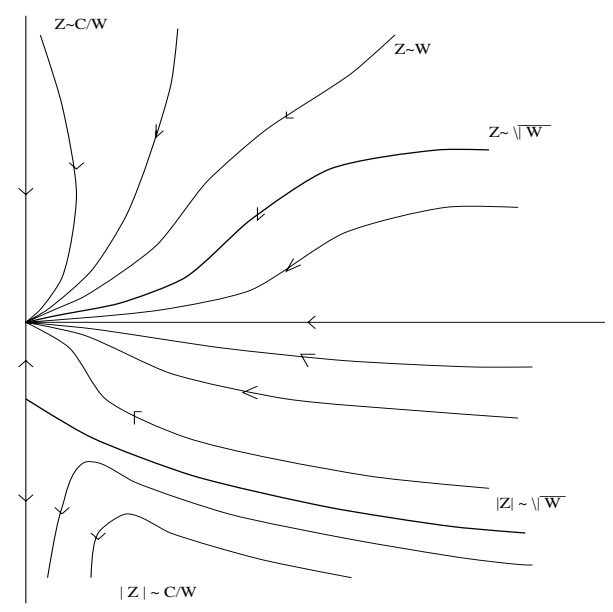

FIG. C.1. Phase portrait.

To draw this phase portrait, it is convenient to introduce a new time variable $\sigma$ defined by $d \sigma=\frac{d \tau}{W}$. In this way, the line $W=0$ becomes a solution of (C.5), (C.6) formed by two stationary points $(0,0),(0,-1)$ and some trajectories connecting them. Another explicit solution is the trajectory corresponding to $Z=0$.

In the region $Z>0$ all the trajectories approach $(0,0)$ as $\tau \rightarrow \infty$ but they have three different behaviors as $\tau \rightarrow-\infty$. More precisely, for some of them $Z \sim \frac{C_{1}}{W}$ as 
$\tau \rightarrow-\infty$ and $W \rightarrow 0$ as $\tau \rightarrow-\infty$. There is another group with the asymptotics $Z \sim C_{2} \sqrt{W}$ and $W \rightarrow \infty$ as $\tau \rightarrow-\infty$. Both groups are separated by a unique trajectory with the asymptotics $Z \sim W$ and $W \rightarrow \infty$ as $\tau \rightarrow-\infty$.

Near $(W, Z)=(0,0)$ the trajectories with $W>0$ behave like

$$
Z \sim k e^{-\frac{1}{2 W}} \quad \text { as } W \rightarrow 0
$$

It is not hard to check that the asymptotics (C.7) imply in the original set of variables (C.3) with $C<0$.

On the other hand, in the $G, \eta$ variables trajectories with the asymptotics $Z \sim \frac{C_{1}}{W}$ provide solutions which vanish at a finite value $\eta_{0}<0$ verifying as $\eta \rightarrow \eta_{0}^{-}$

$$
G \sim C_{1}\left(\eta_{0}-\eta\right)^{\frac{1}{2}} .
$$

The trajectory with the asymptotics $Z \sim W$ provides a solution of (C.1) such that

$$
G \sim k \eta \quad \text { as } \eta \rightarrow 0^{-} .
$$

Finally, trajectories with the asymptotics $Z \sim C_{2} \sqrt{W}$ yield solutions of (C.1) such that

$$
G \sim A^{2}-C A \eta
$$

where $A$ is a positive constant which depends on the starting time for the trajectory.

In the region $Z<0$, a trajectory either reaches $(0,0)$ as $\tau \rightarrow \infty$ or

$$
Z \rightarrow-\frac{C_{1}^{\prime}}{W}, \quad W \rightarrow 0
$$

as $\tau \rightarrow \infty$ or is a separatrix trajectory which reaches $(0,-1)$ as $\tau \rightarrow \infty$. The asymptotics of the separatrix are given by $Z \sim-1-\frac{1}{2} W$ as $\tau \rightarrow \infty$. All the trajectories in this region $Z<0$ satisfy for some $C_{2}^{\prime}$

$$
Z \sim-C_{2}^{\prime} \sqrt{W}, \quad W \rightarrow \infty
$$

as $\tau \rightarrow-\infty$.

The trajectories that approach $(0,0)$ in $Z<0$ yield a solution $G$ satisfying as $\eta \rightarrow \infty$

$$
G \sim C_{3}+C_{4} \frac{e^{-\frac{\eta^{2}}{2 k}}}{|\eta|}
$$

(compare with (C.3)). The separatrix trajectory approaching $(0,-1)$ provides a solution $G$ vanishing at a finite $\eta_{0}>0$ in the form $G \sim C_{5}\left(\eta_{0}-\eta\right)$ as $\eta \rightarrow \eta_{0}^{-}$. Finally, the trajectories satisfying (C.12) provide solutions $G$ with the behavior as $\eta \rightarrow 0^{+}$

$$
G \sim A^{\prime 2}-A^{\prime} C_{2}^{\prime} \eta
$$

Selecting $A^{\prime}=A$ in (C.10), (C.14) (something that can always be achieved by rescaling the solutions) it follows that the trajectories with $C_{2}^{\prime}=C_{2}$ provide a solution to (C.1) which is global and analytic in the whole line.

As we explained in section 5 solutions satisfying the asymptotics (C.9) are not valid to analyze the long time asymptotics of (5.33)-(5.35) with (5.36). Whence, the only valid trajectory is the one with linear behavior at $\eta_{0}>0$, that is, the separatrix indicated in Figure C.1. 


\section{REFERENCES}

[1] E.C. Aifantis, On the problem of dislocation patterning and persistent slip bands, Solid Phenomena State, Vols. 3 and 4 (1988), pp. 397-406.

[2] D.G. Aronson, Nonnegative solutions of linear parabolic equations, Ann. Scuola. Norm. Sup. Pisa, 22 (1968), pp. 607-694.

[3] D.J. Bacon And D. Hull, Introduction to Dislocations, International series on Materials Science and Technology 37, Pergamon Press, Elmsford, NY, 1984.

[4] Z.S. Basinski, M.S. Duesbery, And R. TAYlor, Influence of shear stress on screw dislocations in a model sodium lattice, Canad. J. Phys., 49 (1971), pp. 2160-2180.

[5] A. Carpio and S.J. Chapman, On the modelling of instabilities in dislocation interaction, Philos. Magazine B, 78 (1998), pp. 155-157.

[6] A. Carpio, S.J. Chapman, S.J. Howison, And J.R. OCkendon, Dynamics of line singularities, Philos. Trans. Roy. Soc. London Ser. A, 355 (1997), pp. 2013-2024.

[7] B. Devincre And L.P. Kubin, Simulations of forest interactions and strain hardening in fcc crystals, Model. Simul. Mater. Sci. Eng., 2 (1994), pp. 559-570.

[8] A. Fasano, M. Primicerio, and A.A. Lacey, New results on some classical free boundary problems, Quart. Appl. Math., 38 (1981), pp. 439-460.

[9] A. Friedman, Partial Differential Equations of Parabolic Type, Prentice-Hall, Englewood Cliffs, NJ, 1964.

[10] W.G. Johnston, J.J. Gilman, Dislocation velocities, dislocation densities and plastic flow in lithium fluoride crystals, J. Appl. Phys., 30 (1958), p. 129.

[11] A.K. Head, S.D. Howison, J.R. Ockendon, And S.P. Tighe, An equilibrium theory of dislocation continua, SIAM Rev., 35 (1993), pp. 580-609.

[12] J.P. HiRth, And J. Lothe, Theory of Dislocations, John Wiley and Sons, New York, 1982.

[13] N.M. Kubin, Y. Estrin, And G. Canova, Dislocation patterns and plastic instabilities, in Patterns, Defects and Materials Instabilities, NATO Adv. Sci. Inst. Ser., D. Walgraef and N.M. Ghoniem, eds., Kluwer, Dordrecht, The Netherlands, 1990, pp. 277-301.

[14] P.S. Lomdahl and D.J. Srolovitz, Dislocation generation in the two dimensional FrenkelKontorova model at high stresses, Phys. Rev. Lett., 57 (1986), pp. 2702-2705.

[15] J. SMOLLER, Shock Waves and Reaction-Diffusion Equations, Springer, New York, Berlin, 1983.

[16] M. SheARER AND D. SChAEFFER, The influence of material nonuniformity preceding shear-band formation in a model for granular flow, European J. Appl. Math., 8 (1998), pp. 457-483.

[17] V. VITEK, Effect of dislocation core structure on the plastic properties of metallic materials, in Dislocations and Properties of Real Materials, The Institute of Metals, London, 1985, pp. $30-50$.

[18] G.B. Whitham, Linear and Nonlinear Waves, Wiley Science, New York, London, Sydney, 1974. 